\title{
Dihydrofurocoumarinones - new useful intermediates for substituted and condensed furocoumarins
}

\author{
Valery F. Traven \\ Department of Organic Chemistry, D. Mendeleev University of Chemical Technology of Russia \\ Miusskaja sq.9, Moscow, 125047, Russia \\ Phone: 095-978-94-07. Fax: 095-200-42-04 \\ E-mail:mtraven@main-gw.muctr.edu.ru
}

(received 02 May 00; accepted 21 Sep 00; published on the web 29 Sep 00)

\begin{abstract}
Survey of new synthetic paths to substituted and condensed furocoumarins, perspective compounds for photochemotherapy is given. These furocoumarin syntheses are based on the use of dihydrofurocoumarinones as convenient synthons. Both dihydrofuro[2,3-h]coumarin-9-ones and dihydrofuro[2,3-g]coumarin-6-ones became available via the unusual Fries rearrangement of 7-hydroxycoumarin chloroacetates. Substitution and keto-enol reactions of dihydrofurocoumarinones followed by aromatization of dihydrofuranone moiety are key steps of substituted and condensed furo[2,3-h]- and furo[2,3-g]coumarins synthesis.

We have already characterized in our previous papers many of the compounds listed in the survey. The structures of new compounds are proved by mass spectral, 1H NMR spectral and elemental analysis data
\end{abstract}

Keywords: Dihydrofurocoumarinones, furocoumarins, Fries rearrangement, dihydrofuranone aromatization. 


\section{Introduction}

Furocoumarin derivatives, natural photosensitive compounds have been found to be useful for medical treatment of skin diseases [1,2]. Furocoumarin/ultraviolet therapy, known as photopheresis, has recently been used for treatment of cutaneous T cell lymphoma, Sezary syndrome and related diseases $[3,4]$. Photochemotherapeutic effect of furocoumarins is based on intercalation of the furocoumarin molecule between pyrimidine bases of the microorganism DNA. The intercalation is then followed by the UV light activated cycloaddition reactions of furocoumarin with pyrimidine bases. These [2+2] photocycloaddition reactions provide thus a cross-link of DNA and prevent a microorganism reproduction.

Psoralens, linear furocoumarins have two active sites in the [2+2] photocycloaddition reactions: pyrone ring and furan ring double bonds. This kind difunctionality of furocoumarins has been suggested to be a reason of undesirable side effects of their photoreactions with DNA, which lead to mutagenicity and carcinogenicity[5].

Angelicins, angular furocoumarins show monofunctional properties in their interaction with DNA. They have the only reactive double bond - pyrone ring one. [2+2] Photocycloaddition reactions with furan double bond have been considered as the hindered ones with angelicin derivatives by steric reasons. In spite of their monofunctionality, angelicins possess highly effective phototherapeutic properties. For example, 6,9-dimethyl- and 4,6,9-trimethylangelicins have been shown to be more reactive than psoralens [6-11]. Moreover, angular furocoumarins do not show undesirable side effects and do not have skin toxicity as well [2]. Therefore, syntheses of new psoralen and angelicin derivatives have been intensively studied along last years for purposes of photochemotherapy [[12-19].

We have earlier found dihydrofurocoumarin-9-ones to be very useful intermediates for substituted and condensed furocoumarin synthesis [20-28]. Survey of our previous studies and unpublished results is given in this paper. 


\section{Discussion}

7-Chloroacetoxycoumarins 1a-d undergo unusual Fries rearrangement with dihydrofuro[2,3h]coumarin-9-ones 2a-d formation (Scheme 1)

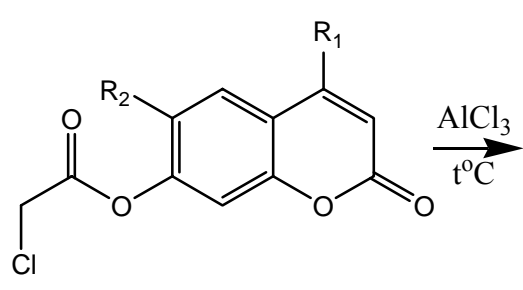

1 a-d<smiles>[R]c1cc2c([R])cc(=O)oc2c2c1OCC2=O</smiles>

2 a-d<smiles>[R]c1cc(=O)oc2cc3c(cc12)C(=O)CO3</smiles>

3 a,b

a: $\mathrm{R}_{1}=\mathrm{R}_{2}=\mathrm{H} ; \mathbf{b}: \mathrm{R}_{1}=\mathrm{Me}, \mathrm{R}_{2}=\mathrm{H} ; \mathbf{c}: \mathrm{R}_{1}=\mathrm{Me}, \mathrm{R}_{2}=\mathrm{Cl} ; \mathbf{d}: \mathrm{R}_{1}=\mathrm{Me}, \mathrm{R}_{2}=\mathrm{Et}$

\section{Scheme 1}

Good yield of dihydrofuro[2,3-h]coumarin-9-ones 2a-d ( up to 70\%) and no any notable amount of dihydrofuro[2,3-g]coumarin-6-ones 3 have been found when the rearrangement was carried out at temperature $115-120^{\circ} \mathrm{C}$.

Nevertheless, linear compounds - dihydrofuro[2,3-g]coumarin-6-ones 5a,b were the predominant products when starting 7-chloroacetoxycoumarins $4 \mathrm{a}$, b contained a substituent at position 8 (Scheme 2) [22]. 
6-Chloroacetyl-7-hydroxy-4-methyl-8-R-coumarins $6 \mathrm{a}, \mathrm{b}$ have also been found in the reaction mixtures. They seem to be the key intermediates in the unusual way of Fries rearrangement of 7hydroxycoumarin chloroacetates.

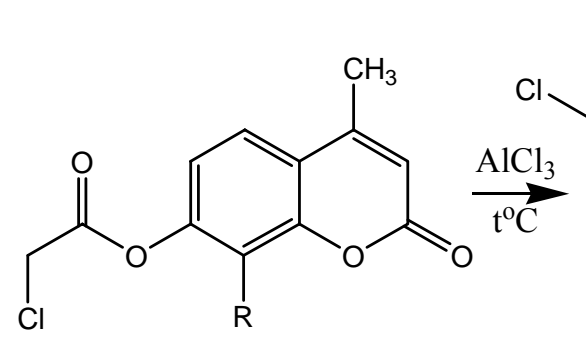

4 a,b
$c_{C}^{C}$

6 a,b<smiles>[R]c1c2c(cc3c(C)cc(=O)oc13)C(=O)CO2</smiles>

5 a,b

$\mathrm{R}: \mathbf{a}, \mathrm{Me} ; \mathbf{b}, \mathrm{Et}$

\section{Scheme 2}

Continuing our studies of these $\mathrm{AlCl}_{3}$-catalyzed reactions we have found conditions when linear cyclizations take place even with substrates which have no any substituents at the position 8 . For example, ratio of linear/angular products of 7-chloroacetoxycoumarins 1a,b rearrangement (Scheme 1) depends upon the reaction temperature in certain extent. Although angular derivatives $2 \mathrm{a}, \mathrm{b}$ are the only products when the rearrangement goes at the temperature $115^{\circ} \mathrm{C}$, yields of linear isomers - dihydrofuro[2,3-g]coumarin-6-ones 3a,b increase (up to 25\%) when reaction temperature is getting higher $\left(\right.$ till $\left.130^{\circ} \mathrm{C}\right)$. Dihydrofuro[2,3-g]coumarin-6-ones $3 \mathrm{a}, \mathrm{b}$ can be isolated and purified by column chromatography and recrystallization. 
Dihydrofuro[2,3-h]coumarin-9-ones 2a, b undergo keto-enol tautomeric transformations (Scheme 3).

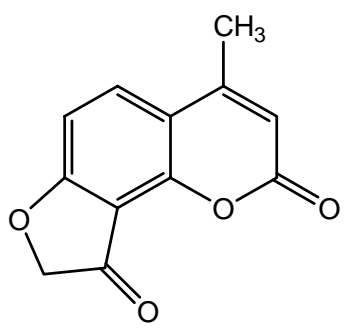

2b, keto

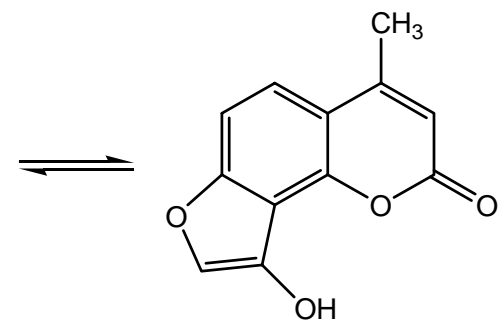

$2 \mathbf{b}$, enol

\section{Scheme 3}

Analyzing 4-methyldihydrofuro[2,3-h]coumarin-9-one $2 b$ reactivity, we have studied its equilibrium transformations in more detail. Electron absorption spectra of $2 \mathrm{~b}$ in $\mathrm{CCl}_{4}-\mathrm{MeOH}$ mixtures have been recorded and an isosbestic point has been registered. Positions of the longestwavelength absorption bands in the UV spectra have been found at $330 \mathrm{~nm}$ in methanol and at $285 \mathrm{~nm}$ in $\mathrm{CCl}_{4}$. We have assigned the band at $330 \mathrm{~nm}$ to the enol form absorption, since its PPP CI-calculated position is located at $328 \mathrm{~nm}$. Both semiempirical and nonempirical quantum chemical calculations showed the keto form to be a predominant one. The difference between formation energies of keto and enol forms has been found equal to -10.57 and $-8.82 \mathrm{kcal} / \mathrm{mol}$ by AM1 and PM3 calculations respectively. 
Keto-enol tautomeric transformation of 4-methyldihydrofuro[2,3-h]coumarin-9-one $2 \mathrm{~b}$ turned to be a key way of many its reactions. We have carried out chlorination and bromination of the compound $2 \mathrm{~b}$ with a good yield [24]. For example, treatment of $2 \mathrm{~b}$ by bromine in acetic acid or 1,4-dioxane provides its mono- and dibromination.

Halogen atoms at position 8 of dihydrofuro[2,3-h]coumarin-9-ones $7 \mathrm{a}, \mathrm{b}$ have been found to be rather reactive in nucleophilic substitution. 8-Bromo-derivative $7 \mathrm{~b}$ has been transformed to 8 hydroxy-, 8-acetoxy- and 8-methoxydihydrofuro[2,3-h]coumarin-9-ones (compounds 7c, 7d, 7e respectively).

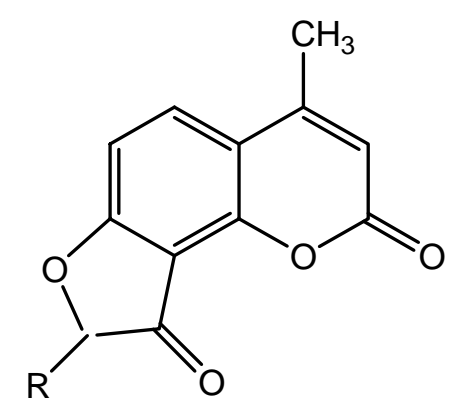

$\mathrm{R}: \mathbf{a}, \mathrm{Cl} ; \mathbf{b}, \mathrm{Br} ; \mathrm{c}, \mathrm{OH} ; \mathbf{d}, \mathrm{OCOMe} ; \mathbf{e}, \mathrm{OMe} ; \mathbf{g}, \mathrm{CHO}$

For example, 8 -hydroxy-derivative $7 \mathrm{c}$ has been prepared by two procedures. Treatment of $7 \mathrm{~b}$ by $\mathrm{AgOH}$ in presence of acetone-water provided 7c formation with $35 \%$ yield[24]. On the other hand, hydrolysis of $7 \mathrm{~d}$ in ethanol-water in presence of $\mathrm{HCl}$ gave semiacetal 8 as a main product. 
Synthesis of 8-formyl-4-methyldihydrofuro[2,3-h]coumarin-9-one $7 \mathrm{~g}$ has been carried out by Vilsmeier-Haack reaction with isolation of dimethylamine 9 as an intermediate product instead of oxonium chloride 10. The following Scheme can be suggested for explanation of the results (Scheme 4).<smiles>CCOC1(O)c2c(ccc3c(C)cc(=O)oc23)OC1O</smiles><smiles>C=C=C1Oc2ccc3c(C)cc(=O)oc3c2C1=O</smiles>

\section{Scheme 4}


In spite of low solubility in many solvents, dimethylamine 9 is easily hydrolyzed in weak acidic solutions with the formyl-derivative $7 \mathrm{~g}$ formation. When hydrolysis has been carried out in presence of concentrated sulfuric acid, decarbonylation of the $7 \mathrm{~g}$ took place with formation of $2 \mathrm{~b}$. The compound $7 \mathrm{~g}$ is also decarbonylated under long heating. The loss of $\mathrm{CO}$ is also a main way of molecular ion fragmentation under mass spectrum recording.

8-Formyl function is the most reactive carbonyl group of the compound $7 \mathrm{~g}$. Its 2,4dinitrophenylhydrazone 11 has been obtained in mild conditions with the quantitative yield.

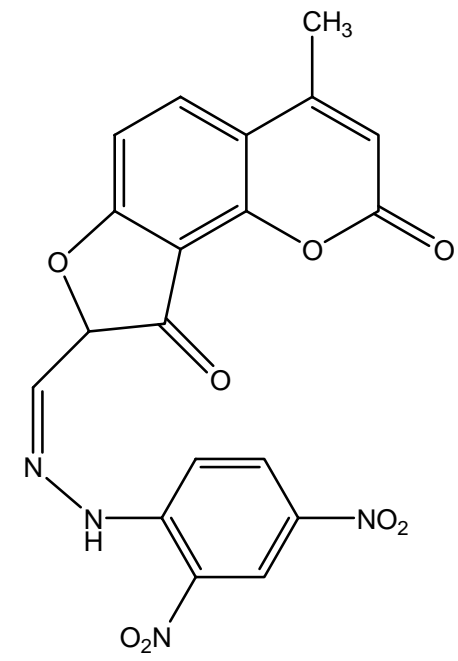

11

4-Methyldihydrofuro[2,3-h]coumarin-9-one $2 \mathrm{~b}$ undergoes easily condensations with aldehydes and ketones in acetic acid in the presence of $\mathrm{HCl}$ (Scheme 5). According to the spectral data, condensation products $12 \mathrm{a}-\mathrm{h}$ have a croton-type structure [24]. 

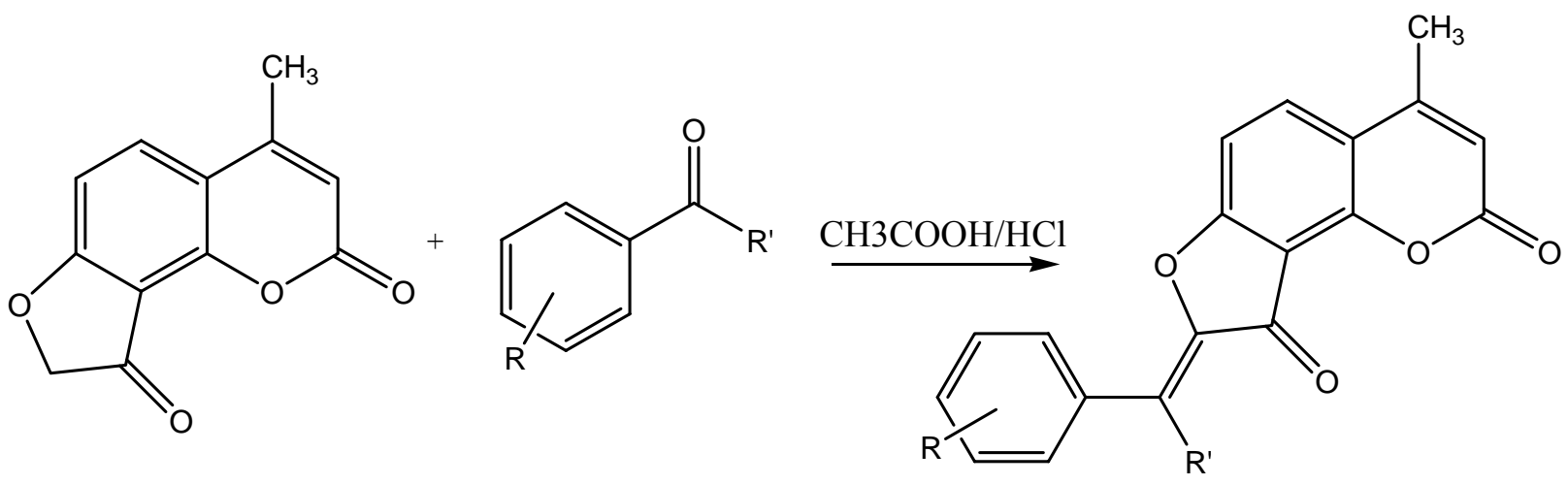

12 a-h

a-g: $\mathrm{R}=\mathrm{H} ; \mathbf{a}, \mathrm{R}+\mathrm{H} ; \mathbf{b}, \mathrm{R}=4-\mathrm{Br} ; \mathbf{c}, \mathrm{R}=4-\mathrm{Me}_{2} \mathrm{~N} ; \mathbf{d}, \mathrm{R}=4-\mathrm{OH}, 3-\mathrm{OMe}$;

e, $\mathrm{R}=2-\mathrm{NO}_{2} ; \mathbf{f}, \mathrm{R}=2-\mathrm{OH} ; \mathbf{g}, \mathrm{R}=4-\mathrm{NO}_{2} ; \mathbf{h}, \mathrm{R}=\mathrm{R}^{\prime}=\mathrm{Me}$

\section{Scheme 5}

Dihydrofuro[2,3-h]coumarin-9-ones undergo azocoupling reactions as well. For example, compound $2 \mathrm{~b}$ reacts with aryldiazonium chlorides in acetic acid in the presence of pyridine with formation of 8-arylazo-4-methyldihydrofuro[2,3-h]coumarin-9-ones 13a-n (Scheme 6). 


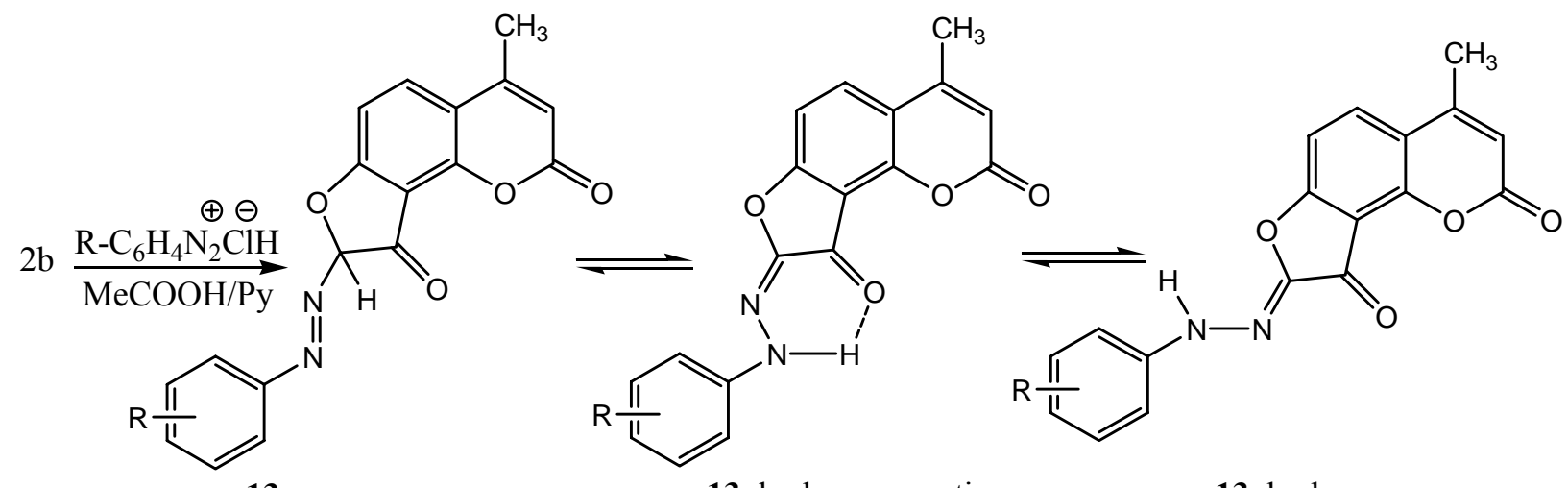

13, azo

13, hydrazone, anti

13, hydrazone, syn

13 a-p: R: a, $\mathrm{H} ; \mathbf{b}, 4-\mathrm{OMe} ; \mathbf{c}, \mathrm{Cl} ; \mathbf{d}, \mathrm{NO}_{2}, \mathbf{e}, 4-\mathrm{Me} ; \mathbf{f}, 4-\mathrm{Cl} ; \mathrm{g}, 2-\mathrm{NO}_{2} ; \mathbf{h}, 2-\mathrm{NO}_{2}-4-\mathrm{NO}_{2} ; \mathbf{i}, 4-\mathrm{NEt}_{2}$; j, 2-Et; k, 3-Et; l, 4-Et; m, 2-Me-3-Me; n, 2-Me; o, 4-Br; $\mathbf{p}, 3-\mathrm{NO}_{2}$

\section{Scheme 6}

We have studied azo-quinone hydrazone tautomeric transformations of the compounds 13 with use of electron absorption spectra and quantum chemical calculations, both semiempirical and nonempirical. Hydrazone tautomeric forms have been found to be the predominant ones. For example, a good correlation between calculated by the PPP CI method and experimental positions $(\lambda \max )$ of the longest-wavelength absorption bands has been found when anti-isomer of quinone hydrazone form used as a model in the calculations [25] (Table 1).

Calculated by the PPP CI method and experimental positions ( $\lambda$ max) of the longest-wavelength absorption bands of the compounds 13 a-d.

N-H Singlet signal at 11.0-11.3 ppm in the 1H NMR spectra and characteristic peaks in the mass spectra approve quinone hydrazone tautomeric form of the azocompounds 13. NH-Function in the compounds 13 has been acetylated [25] with formation of $\mathrm{N}$-acetyl quinone hydrazones 14. 


\section{Table 1}

\begin{tabular}{lllll}
\hline Compd number & $\mathrm{R}$ & $\lambda$ max,exp. & $\begin{array}{l}\lambda \max , \text { antiform } \\
\text { (calculated) }\end{array}$ & $\begin{array}{l}\lambda \text { max,syn form } \\
\text { (calculated) }\end{array}$ \\
\hline $13 \mathrm{a}$ & $\mathrm{H}$ & 428 & 429 & 392 \\
$13 \mathrm{~b}$ & $\mathrm{OMe}$ & 451 & 444 & 405 \\
$13 \mathrm{c}$ & $\mathrm{Cl}$ & 430 & 432 & 396 \\
$13 \mathrm{~d}$ & $\mathrm{NO} 2$ & 431 & 433 & 394 \\
\hline
\end{tabular}

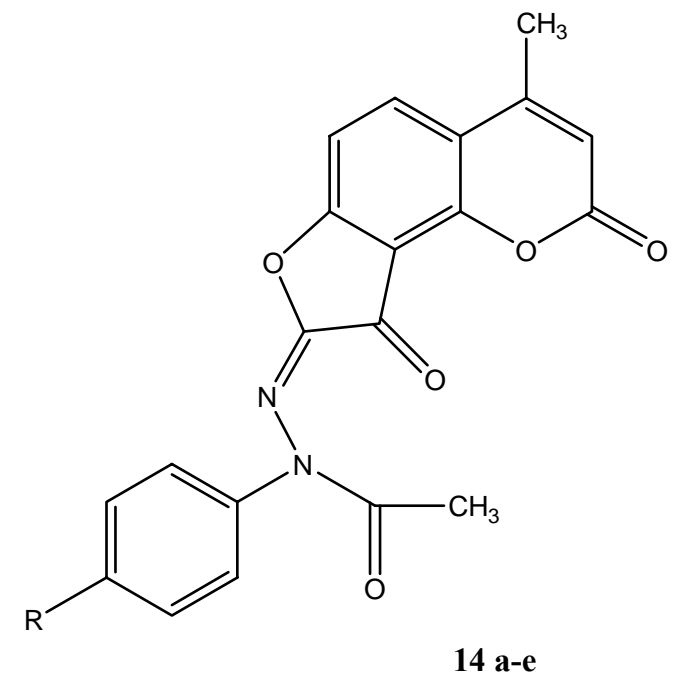

R: a, H; b, 4-OMe; c, 4-Br; d, 4-Cl; e, 4-Me

Dihydrofuro[2,3-h]coumarin-9-ones can also undergo azocoupling reactions at position 6. It happens when reactions with aryldiazonium tetrafluoroborates are carried out in the presence of sodium hydroxide. For example, R-substituted phenyldiazonium tetrafluoroborates and $2 \mathrm{~b}$ react with formation of azocoupling products 15a-e, 4-methyl-6-(R-phenylazo)dihydrofuro[2,3h] coumarin-9-ones with a very good yield. The regioselectivity is due to lactone ring opening in 
the presence of strong base. The formed phenoxide ion (Scheme 7) reacts with electrophiles at position 6 .

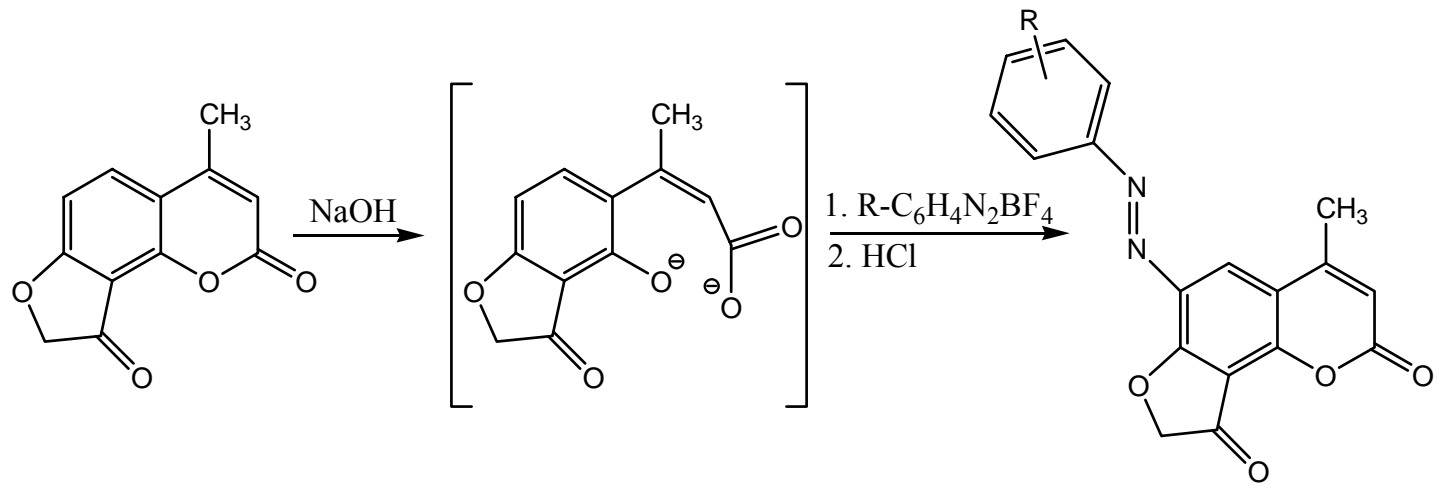

R: a, H; b, 4-OMe; c, 4-NO ${ }_{2}$; d, 3-Et; e, 4-NMe 215 a-e

\section{Scheme 7}

Resonance stabilization of the intermediate $\sigma$-complex is much facilitated along azocoupling reaction with the lactone open form. The final acidification recovers cyclic form of coumarin moiety with 6-arylazo-4-methyldihydrofuro[2,3-h]coumarin-9-ones 15a-e formation. 


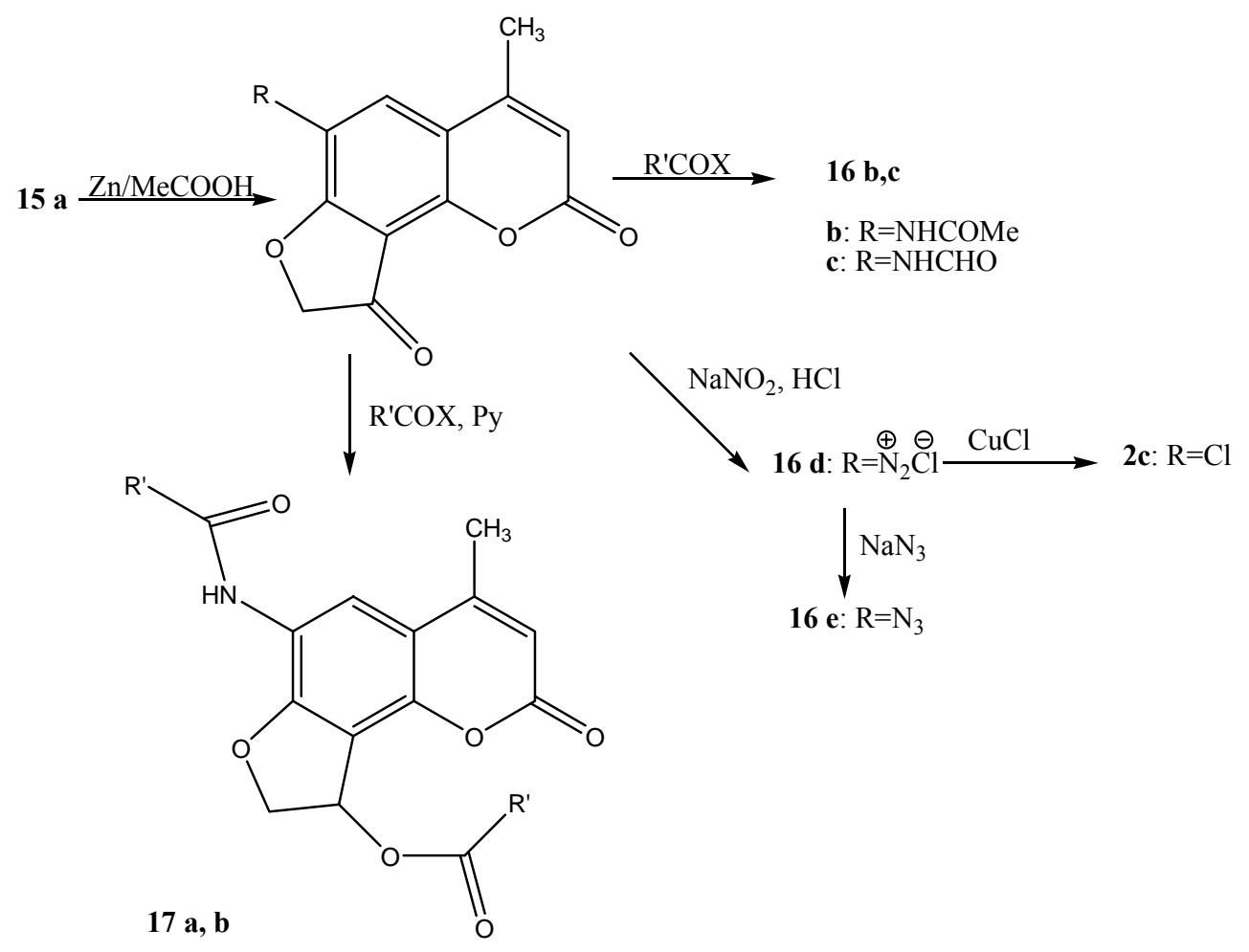

a: $\mathrm{R}^{\prime}=\mathrm{Me} ; \mathbf{b}: \mathrm{R}^{\prime}=\mathrm{Ph}$

\section{Scheme 8}

On opposite to the compounds 13a-n, azocoupling products 15a-e possess an azo tautomeric form. Therefore, compounds 15a-e has been easily reducted. For example, reduction of azo function in azocompound 15a leads to 6-amino-4-methyldihydrofuro[2,3-h]coumarin-9-one 16a with a very good yield. Transformations of the amino function in it, followed by aromatization of the dihydrofuranone ring provides a series of new angelicin derivatives [27] (Scheme 8).

For example, amino function of $16 \mathrm{a}$ is readily acylated. Thus, treatment of $16 \mathrm{a}$ by acetic anhydride under heating provides 6-acetamido-4-methyldihydrofuro[2,3-h]coumarin-9-one 16b formation. Treatment of 16a by acetic anhydride in presence of pyridine provides both $\mathrm{N}$ - and $\mathrm{O}$ acetylation with formation of 6-acetamido-9-acetoxy-4-methylangelicin 17a. Similar, benzoylation of $16 \mathrm{a}$ by benzoyl chloride in presence of pyridine gives 6-benzamido-9benzoyloxy-4-methylangelicin $17 \mathrm{~b}$. Heating of $16 \mathrm{a}$ in formic acid gives 6-formamido-4methyldihydrofuro[2,3-h] coumarin-9-one $16 \mathrm{c}$. 
Amine 16a undergoes diazotization under treatment with sodium nitrite and hydrochloric acid. The product of diazotization, 4-methyldihydrofuro[2,3-h]coumarin-9-one-6-diazonium chloride $16 \mathrm{~d}$ transforms to 6-chloro-4-methyldihydrofuro[2,3-h]coumarin-9-one 16e via the Sandmeyer reaction and to 6-azido-4-methyldihydrofuro[2,3-h]coumarin-9-one $16 \mathrm{f}$ under treatment with sodium azide.

Both 4-methyldihydrofuro[2,3-h]coumarin-9-ones 2a, b (unsubstituted in furanone and benzene rings) and their 8- and 6-substituted derivatives undergo rather easily transformation to the proper angelicins. That kind of transformation has been done by two procedures: 1) reduction of furanone moiety followed by dehydration of the resulted alcohols 18 into angelicins 19 as a final products; 2) O-acetylation of dihydrofuranone enol form with formation of 9-acetoxyangelicins 20. Alcohols 18, angelicins 19, and 9-acetoxyangelicins 20 are listed on the Scheme 9.

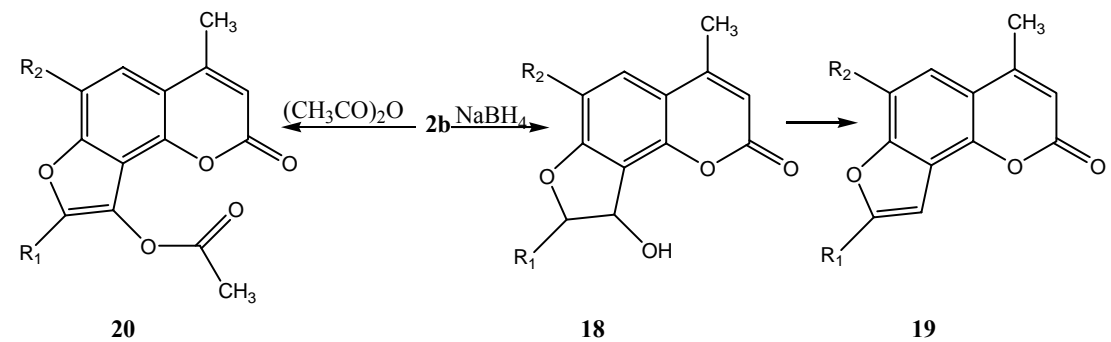

\section{Scheme 9}




\begin{tabular}{|c|c|c|}
\hline \multirow[t]{9}{*}{ 18: } & a: $\mathrm{R}_{1}=\mathrm{H} ; \mathrm{R}_{2}=\mathrm{Cl}$ & 19: $\mathbf{a}: \mathrm{R}_{1}=\mathrm{H} ; \mathrm{R}_{2}=\mathrm{Cl}$ \\
\hline & b: $\mathrm{R}_{1}=\mathrm{H} ; \mathrm{R}_{2}=\mathrm{Et}$ & b: $\mathrm{R}_{1}=\mathrm{H} ; \mathrm{R}_{2}=\mathrm{Et}$ \\
\hline & c: $\mathrm{R}_{1}=\mathrm{H} ; \mathrm{R}_{2}=4-\mathrm{Me}_{2} \mathrm{~N}-\mathrm{C}_{6} \mathrm{H}_{4} \mathrm{~N}_{2}$ & c: $\mathrm{R}_{1}=\mathrm{H} ; \mathrm{R}_{2}=3,5-\mathrm{Me}_{2} \mathrm{C}_{3} \mathrm{HN}_{2}$ \\
\hline & d: $\mathrm{R}_{1}=\mathrm{H} ; \mathrm{R}_{2}=\mathrm{NH}_{2}$ & d: $\mathrm{R}_{1}=\mathrm{H} ; \mathrm{R}_{2}=\mathrm{NH}_{2}$ \\
\hline & e: $\mathrm{R}_{1}=\mathrm{H} ; \mathrm{R}_{2}=\mathrm{NHCOCH}_{3}$ & e: $\mathrm{R}_{1}=\mathrm{H} ; \mathrm{R}_{2}=\mathrm{CH}_{3} \mathrm{CONH}$ \\
\hline & $\mathbf{f}: \mathrm{R}_{1}=\mathrm{H} ; \mathrm{R}_{2}=\mathrm{NHCHO}$ & f: $\mathrm{R}_{1}=\mathrm{H} ; \mathrm{R}_{2}=\mathrm{NHCHO}$ \\
\hline & g: $\mathrm{R}_{1}=\mathrm{H} ; \mathrm{R}_{2}=\mathrm{N}_{3}$ & g: $\mathrm{R}_{1}=\mathrm{H} ; \mathrm{R}_{2}=\mathrm{N}_{3}$ \\
\hline & b: $\mathrm{R}_{1}=\mathrm{H} ; \mathrm{R}_{2}=\mathrm{Et}$ & h: $\mathrm{R}_{1}=\mathrm{H} ; \mathrm{R}_{2}=4-\mathrm{CH}_{3}-\mathrm{C}_{6} \mathrm{H}_{4} \mathrm{SO}_{2} \mathrm{NH}$ \\
\hline & & i: $\mathrm{R}_{1}=\mathrm{H} ; \mathrm{R}_{2}=4-\mathrm{OH}-\mathrm{C}_{6} \mathrm{H}_{4} \mathrm{CHN}$ \\
\hline \multirow[t]{10}{*}{ 20: } & $\mathbf{a}: \mathrm{R}_{1}=\mathrm{R}_{2}=\mathrm{H}$ & $\mathbf{j}: \mathrm{R}_{1}=\mathrm{H} ; \mathrm{R}_{2}=\mathrm{NHNH}_{2}$ \\
\hline & b: $\mathrm{R}_{1}=\mathrm{H} ; \mathrm{R}_{2}=\mathrm{Et}$ & $\mathbf{k}: \mathrm{R}_{1}=\mathrm{H} ; \mathrm{R}_{2}=4-\mathrm{O}_{2} \mathrm{~N}-\mathrm{C}_{6} \mathrm{H}_{4}-\mathrm{CHNNH}$ \\
\hline & c: $\mathrm{R}_{1}=\mathrm{Cl} ; \mathrm{R}_{2}=\mathrm{H}$ & I: $\mathrm{R}_{1}=\mathrm{H} ; \mathrm{R}_{2}=4-\mathrm{HO}-\mathrm{C}_{6} \mathrm{H}_{4}-\mathrm{CHNNH}$ \\
\hline & d: $\mathrm{R}_{1}=\mathrm{Br} ; \mathrm{R}_{2}=\mathrm{H}$ & $\mathbf{m}: \mathrm{R}_{1}=\mathrm{H} ; \mathrm{R}_{2}=4-\mathrm{Me}_{2} \mathrm{~N}-\mathrm{C}_{6} \mathrm{H}_{4}-\mathrm{N}_{2}$ \\
\hline & e: $\mathrm{R}_{1}=\mathrm{H} ; \mathrm{R}_{2}=\mathrm{PhN}=\mathrm{N}-$ & \\
\hline & f: $\mathrm{R}_{1}=\mathrm{H} ; \mathrm{R}_{2}=4-\mathrm{MeO}-\mathrm{C}_{6} \mathrm{H}_{4}-\mathrm{N}=\mathrm{N}-$ & \\
\hline & g: $\mathrm{R}_{1}=\mathrm{H} ; \mathrm{R}_{2}=4-\mathrm{NO}_{2}-\mathrm{C}_{6} \mathrm{H}_{4}-\mathrm{N}=\mathrm{N}-$ & \\
\hline & $\mathbf{h}: \mathrm{R}_{1}=\mathrm{COMe} ; \mathrm{R}_{2}=\mathrm{H}$ & \\
\hline & i: $\mathrm{R}_{1}=\mathrm{COMe} ; \mathrm{R}_{2}=\mathrm{NHCHO}$ & \\
\hline & $\mathbf{j}: \mathrm{R}_{1}=\mathrm{H} ; \mathrm{R}_{2}=\mathrm{NHCHO}$ & \\
\hline
\end{tabular}

There are some comments to the acetylation procedure. When acetylation is carried out by acetic anhydride in the presence of potassium acetate, 9-acetoxy-derivatives 20 are the final products. When compound $2 \mathrm{~b}$ is treated by acetic anhydride in presence of sulfuric acid or pyridine, both $\mathrm{O}$-acetylation and $\mathrm{C}$-acetylation take place with formation of diacetyl-derivative $20 \mathrm{~h}$.

Acetylation of $7 \mathrm{~g}$ provides rather an unusual result. Dimer 21 has been isolated as a main product. The following steps of aldehyde $7 \mathrm{~g}$ transformation can be suggested: $7 \mathrm{~g}$ undergoes partly decarbonylation along the acetylation procedure. The formed amount of $2 \mathrm{~b}$ reacts then with the starting aldehyde $7 \mathrm{~g}$ via croton condensation with formation of 21 (Scheme 10). 


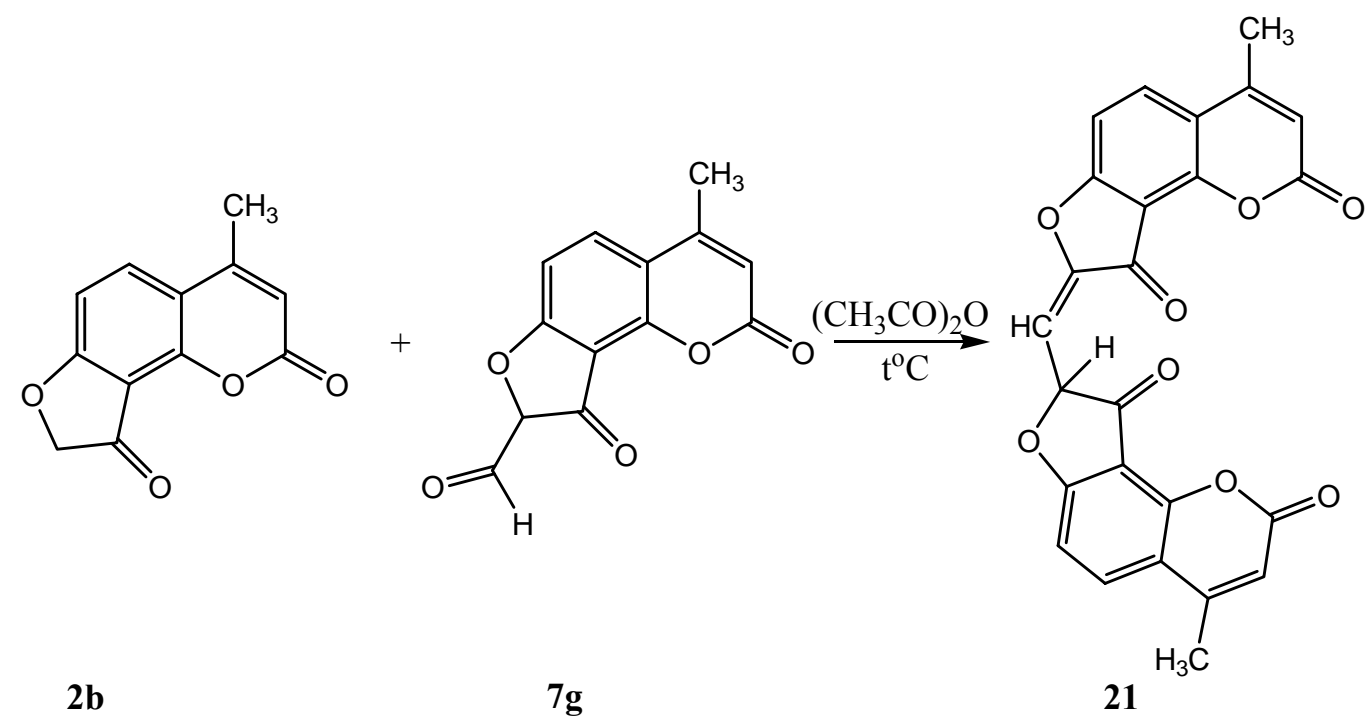

\section{Scheme 10}

Linear dihydrofuro[2,3-g]coumarin-6-ones 3 and 5 seem to undergo keto-enol transformations as well. Nevertheless an isosbestic points have not been seen in their electron absorption spectra when $\mathrm{CCl}_{4}-\mathrm{MeOH}$ mixtures are used as solvents. We have found the differences between formation energies of keto and enol tautomeric forms of $3 b$ to be much higher, when compared with that of the $2 \mathrm{~b}$ tautomeric forms: -15.72 and $-12.57 \mathrm{kcal} / \mathrm{mol}$ by AM1 and PM3 calculations respectively. Keto form of $3 \mathrm{~b}$ turns to be relatively more stable one. Nevertheless dihydrofuro[2,3-g]coumarin-6-ones seem to be similar in reactivity to dihydrofuro[2,3h]coumarin-9-ones.

Linear dihydrofuro[2,3-g]coumarin-6-ones undergo also transformation to psoralen derivatives. Compounds $3 \mathrm{~b}$ and $5 \mathrm{a}$ have been transformed into psoralens 22 and 23 by reduction/dehydration and acetylation procedures respectively (Scheme 11). 
<smiles></smiles>

23: $\mathrm{R}_{1}=\mathrm{R}_{2}=\mathrm{Me}$

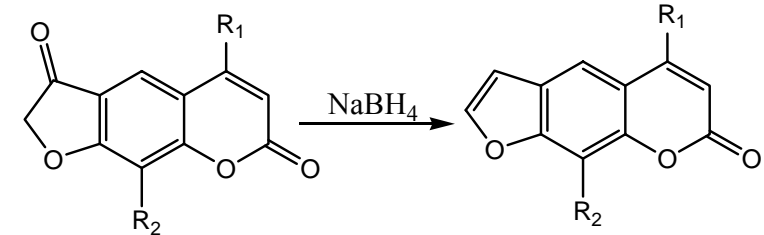

3b: $\mathrm{R}_{1}=\mathrm{Me}, \mathrm{R}_{2}=\mathrm{H}$ 5a: $\mathrm{R}_{1}=\mathrm{R}_{2}=\mathrm{Me}$

22: $\mathrm{R}_{1}=\mathrm{Me}, \mathrm{R}_{2}=\mathrm{H}$

\section{Scheme 11}

4-Methyldihydrofuro[2,3-g]coumarin-6-one undergoes an azocoupling reaction at position 7 in the presence of weak acid. In accordance with spectral data, 4-methyl-6-(4'metoxyphenylazo)dihydrofuro[2,3-g]coumarin-6-one 24 possesses an quinone hydrazone tautomeric form.

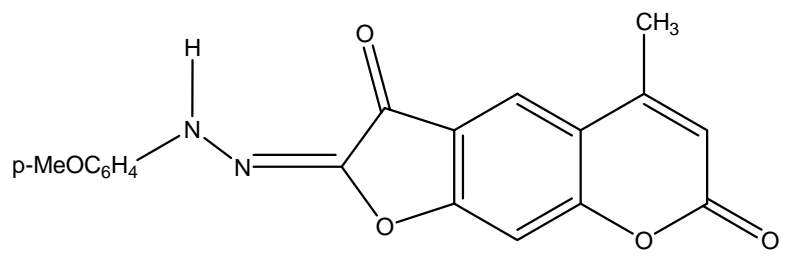

24

Diazocompound $16 \mathrm{~d}$ has been reducted with formation of hydrazine derivative 25. Hydrazine 25 is sensitive to heating. Its preparation goes with a good yield if temperature of the reaction is below $-10^{\circ} \mathrm{C}$. If temperature of the reduction is higher, the reaction is accompanied by formation of amine $16 \mathrm{a}$ and traces of coumarinone $2 \mathrm{~b}$.

Hydrazine 25 reacts readily with benzaldehydes [27]. However, regioselectivity of these reactions depends also upon the temperature. For example, hydrazone 26 has smoothly been 
prepared along condensation of 25 with 4-fluorobenzaldehyde in acetic acid at temperature $\mathrm{O}^{\circ} \mathrm{C}$. At higher temperature condensation with 4-fluorobenzaldehyde takes place also at position 8 (methylene group of dihydrofuranone ring). Bis-benzylidene derivative 27 is a product of the reaction (Scheme 12).

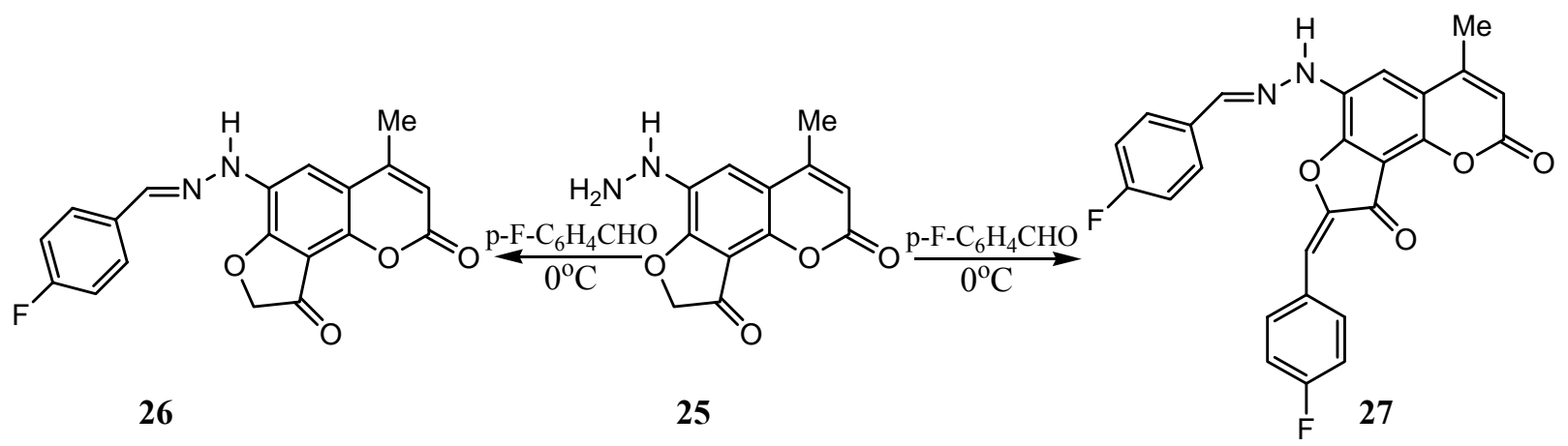

\section{Scheme 12}

Position 8 seems to be rather reactive in the amine 16a. If condensations of amine 16a with benzaldehyde, pentafluorobenzaldehyde and 3,4,5-tris(methoxy)benzaldehyde take place in acetic acid in the presence of concentrated hydrochloric acid, reactions go at the position 8 only (leaving 6-amino function untouched) with formation of C-benzylidene derivatives 28a-c respectively.

These derivatives are analogues of C-benzylidene derivatives, which we have earlier studied in more detail [24]. C-H-Bond seems to be more nucleophilic in these conditions than the 6amino function.

Moreover, pentafluorobenzaldehyde, the most active benzaldehyde reacts only at the position 8 of $16 \mathrm{a}$ in the presence of HCI. One can suggest, N-protonation decreases concentration of the active form of amin 16a. Benzylidene derivative 28a undergoes easily an acetylation and formylation in standard procedures with formation of compounds 29a, b respectively (Scheme $13)$. 
Diazotization of 6-amino-4-methylangelicin 19, followed by reduction of the resulted diazocompound gave 6-hydrazino-4-methylangelicin 30. This derivative turned to become as a key compound in new pyrrolo[2,3-f]furo[2,3-h]coumarins synthesis via indole cyclization by Fisher [26]. The respective hydrazones 31,33, 35 and pyrrolo[2,3-f]furo[2,3-h]coumarins 32, 34 and 36 are listed below on the Scheme 14 .<smiles>Cc1cc(=O)oc2c3c(c(N)cc12)OCC3=O</smiles>

$16 a$<smiles>CC(=O)Nc1cc2c(C)cc(=O)oc2c2c1O/C(=C\c1ccccc1)C2=O</smiles>

28 a: $\mathrm{Ar}=\mathrm{Ph}$

b: $\mathrm{Ar}=\mathrm{C}_{6} \mathrm{~F}_{5}$

c: $\mathrm{Ar}=3,4,5-(\mathrm{MeO}){ }_{3} \mathrm{C}_{6} \mathrm{H}_{2-}$
29 a: $\mathrm{R}=\mathrm{Me}$

b: $\mathrm{R}=\mathrm{H}$

\section{Scheme 13}




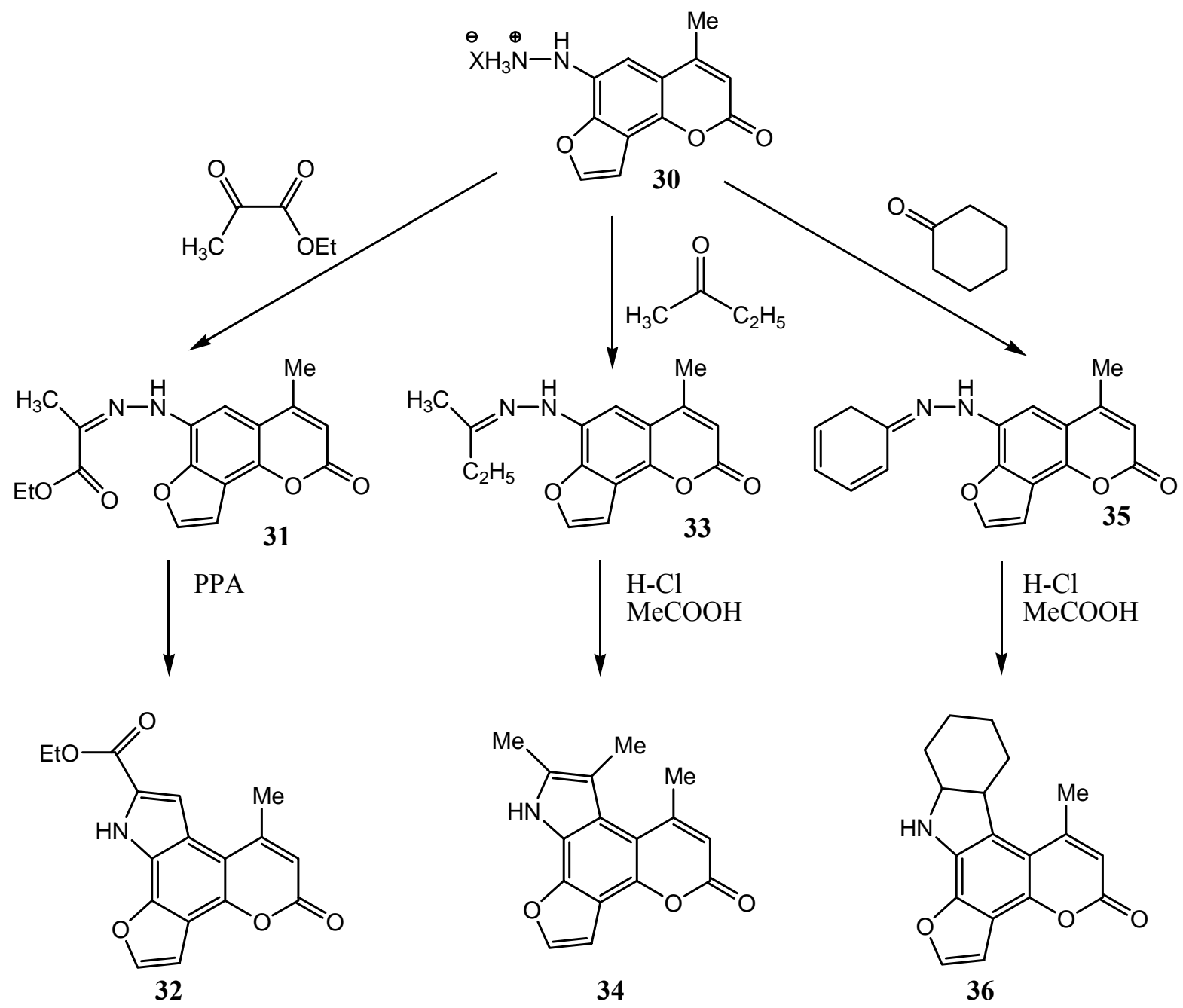

Scheme 14 


\section{Experimental Section}

\section{General Procedures.}

1H NMR spectra were recorded on a Varian Gemini-200 spectrometer at $200 \mathrm{MHz}$ in acetone$\mathrm{d}_{6}$, DMSO- $\mathrm{d}_{6}$ or $\mathrm{CDCI}_{3}$ solutions using TMS as internal standard. Chemical shifts are given in ppm.

Mass spectra were scanned on a SSQ-710 (Finnigan MAT) spectrometer at the energy of ionizing electrons equal to $70 \mathrm{ev}$.

Electron absorption spectra were recorded on a spectrophotometer "Specord-UV/VIS" using $\mathrm{CCl}_{4}$, methanol or ethanol solutions.

Fries rearrangement of 7-chloroacetoxycoumarins 1a-d, 4a,b.

The mixture of 7-chloroacetoxycoumarin 1 or $4(0.016 \mathrm{~mol})$ and anhydrous aluminium chloride $(0.052 \mathrm{~mol})$ was heated in an oil bath at $115-120^{\circ} \mathrm{C}$ for $25-35 \mathrm{~min}$. The cooled reaction mixture was poured into cold dilute $\mathrm{HCl}$. The crude solid, thus obtained, was washed with water and recrystallized from glacial acetic acid. Compounds 2 a-d were thus obtained. The filtrate was evaporated and compound $3 \mathrm{~b}$ was obtained by column chromatography (silica gel 40/100, $\mathrm{CHCl}_{3}$ ) and recrystallization from glacial acetic acid.

\section{6-Chloro-4-methyldihydrofuro[2,3-h]coumarin-9-one (2c).}

yield $=20 \%$, light yellow crystals, mp $244-245^{\circ} \mathrm{C} .1 \mathrm{H} \mathrm{NMR}$ (acetone-d $\mathrm{d}_{6} ; \delta, \mathrm{ppm} ; \mathrm{J}, \mathrm{Hz}$ ): 2.53 (d, 3H, 4-Me, $\mathrm{J}_{\mathrm{Me}, 3}=1.2$ ), 4.96 (s, 2H, $-\mathrm{CH}_{2}-$ ), 6.31 (q, 1H, 3-H, J 3,Me $=1.2$ ), 8.14 (s, 1H, 5-H). MS (m/z, \%): 250/252 (1Cl)(M+, 100), 222/224 (1Cl)(-CO, 84), 193/195 (1Cl), (-CO,-HCO, 59), 149/151 (1Cl)(-2CO,-HCO,17).

Anal. calc. for $\mathrm{C}_{12} \mathrm{H}_{7} \mathrm{O}_{4} \mathrm{Cl}$ : C 57.48, $\mathrm{H}$ 2.79; found: C 57.23, $\mathrm{H} 2.91$. 


\section{6-Ethyl-4-methyldihydrofuro[2,3-h]coumarin-9-one (2d).}

yield $=75 \%$, light yellow crystals, mp $243-244^{\circ} \mathrm{C} .1 \mathrm{H}$ NMR (acetone-d 6 ; $\delta$, ppm; J, Hz): $1.29(\mathrm{t}$, $\left.3 \mathrm{H}, \mathrm{Me}, \mathrm{J}_{\mathrm{Me},-\mathrm{CH} 2-}=7.7\right), 2.50$ (d, 3H, 4-Me, $\left.\mathrm{J}_{\mathrm{Me}, 3}=1.2\right), 2.76$ (q, 2H, - $\left.\mathrm{CH}_{2^{-}}, \mathrm{J}_{-\mathrm{CH} 2-\mathrm{Me}}=7.7\right), 4.85$ (s, 2H, $-\mathrm{CH}_{2^{-}}$), 6.21 (q, 1H, 3-H, J $\left.3, \mathrm{Me}=1.2\right), 7.91(\mathrm{~s}, 1 \mathrm{H}, 5-\mathrm{H})$.

MS (m/z, \%): $244(\mathrm{M}+, 100), 216$ (-CO, 85), 187 (-CO, - HCO, 20), 229 (-Me, 79), 201 (-Me, $\mathrm{CO}, 98), 173(-\mathrm{Me},-2 \mathrm{CO}, 26)$.

Anal. calc. for $\mathrm{C}_{14} \mathrm{H}_{12} \mathrm{O}_{4}$ : C 68.85, $\mathrm{H} 4.92$; found: $\mathrm{C} 68.79, \mathrm{H} 4.98$.

\section{4-Methyldihydrofuro[2,3-g]coumarin-9-one (3b).}

yield $=15 \%$, light yellow crystals, mp 234-236 ${ }^{\circ} \mathrm{C} .1 \mathrm{H} \mathrm{NMR}\left(\mathrm{DMSO}-\mathrm{d}_{6}, \delta, \mathrm{ppm} ; \mathrm{J}, \mathrm{Hz}\right): 2.47$ (d, 3H,4-Me, J $\left.\mathrm{Me}, 3_{3}=1.1\right), 4.91\left(\mathrm{~s}, 2 \mathrm{H}, \mathrm{CH}_{2}\right), 6.34$ (q, 1H, 3-H, J 3,Me $\left.=1.1\right), 7.28(\mathrm{~s}, 1 \mathrm{H}, 9-\mathrm{H}), 8.05$ (s, $1 \mathrm{H}, 5-\mathrm{H})$.

MS (m/z, \%): $216(\mathrm{M}+, 100), 188(-\mathrm{CO}, 52), 159$ (-CO,-HCO, 59), 131 (-2CO, -HCO,18).

Anal. calc. for $\mathrm{C}_{12} \mathrm{H}_{8} \mathrm{O}_{4}$ : C 66.67, $\mathrm{H} 3.73$; found: C 66.79, $\mathrm{H} 3.88$.

\section{8,9-Dihydroxy-9-ethoxy-4-methyl-8,9-dihydrofuro[2,3-h]coumarin (8).}

Mixture of 8-acetoxy-4-methyldihydrofuro[2,3-h]coumarin-9-one $(0.4 \mathrm{~g}, 0.0014 \mathrm{~mol}) 5 \mathrm{ml}$ of ethanol and $5 \mathrm{ml}$ of $\mathrm{HCl}$ stir intensively for $4 \mathrm{hrs}$. at $20^{\circ} \mathrm{C}$ and pour into water. The product was extracted by $\mathrm{CHCl}_{3}$ and purified by column chromatography (silica gel 40/100, $\mathrm{CHCl}_{3}$ ), compound 8 , yield $=10 \%$, white crystals, mp $155-156^{\circ} \mathrm{C}$. $1 \mathrm{H}$ NMR (acetone- $\mathrm{d}_{6} ; \delta$, ppm; J, Hz): $1.16\left(\mathrm{t}, 3 \mathrm{H}, \mathrm{Me}, \mathrm{J}_{\mathrm{Me},-\mathrm{CH} 2-}=7.4\right), 2.43$ (d, 3H, 4-Me, J Me,3 $\left.=1.2\right), 4.18$ (q, 2H, - $\mathrm{CH}_{2^{-}}, \mathrm{J}_{-\mathrm{CH} 2-\mathrm{Me}}$ =7.4), $4.96\left(\mathrm{~d}, 1 \mathrm{H}, 8-\mathrm{OH}, \mathrm{J}_{8-\mathrm{OH}, 8-\mathrm{H}}=5.0\right), 5.81\left(\mathrm{~d}, 1 \mathrm{H}, \mathrm{J}_{8-\mathrm{H}, 8-\mathrm{OH}}=5.0\right), 6.11\left(\mathrm{q}, 1 \mathrm{H}, 3-\mathrm{H}, \mathrm{J}_{3, \mathrm{Me}}\right.$ $=1.2), 6.91\left(\mathrm{~d}, 1 \mathrm{H}, 6-\mathrm{H}_{1} \mathrm{~J}_{6,5}=8.9\right), 7.61\left(\mathrm{~d}, 1 \mathrm{H}, 5-\mathrm{H}, \mathrm{J}_{5,6}=8.9\right), 9.54(\mathrm{~s}, 1 \mathrm{H}, 9-\mathrm{OH})$. MS (m/z, \%): $278(\mathrm{M}+, 20), 205$ (- O=C-OEt, 100), $176(-\mathrm{O}=\mathrm{C}-\mathrm{OEt},-\mathrm{HCO}, 6), 260\left(-\mathrm{H}_{2} \mathrm{O}, 5\right)$, $232(-\mathrm{EtOH}, 6)$.

Anal. calc. for $\mathrm{C}_{14} \mathrm{H}_{14} \mathrm{O}_{6}$ : C 60.43, $\mathrm{H}$ 5.07; found: C 60.29, $\mathrm{H}$ 5.07. 
8-( $N, N$-Dimethylaminomethylideno)-4-methyldihydrofuro[2,3-h]coumarin-9-one (9).

Phosphorous oxychloride $(2.3 \mathrm{ml})$ was added drop by drop to DMFA $(2 \mathrm{ml})$ at $10^{\circ} \mathrm{C}$. Solution of compound $2 \mathrm{~b}(4 \mathrm{~g}, 0.018 \mathrm{~mol})$ in $25 \mathrm{ml}$ of DMFA and $75 \mathrm{ml}$ dioxane was added then to the $\mathrm{POCl}_{3}$-DMFA mixture. Reaction mixture was heated then for 2 hrs on water bath. The precipitate was filtered off, washed by water and hot acetone ( 3 times). Compound 9, yield=90\%, yellow crystals, mp 266-267 C. 1H NMR (DMSO-d 6 , $\delta$, ppm; J, Hz): 2.45 (d, 3H, 4-Me, J Me,3 $=1.2), 3.29\left(\mathrm{~s}, 6 \mathrm{H},-\mathrm{N}(\mathrm{Me})_{2}, 6.33\right.$ (q, 1H, 3-H, J $\left.3, \mathrm{Me}=1.2\right), 7.24(\mathrm{~s}, 1 \mathrm{H},=\mathrm{CH}-\mathrm{N}), 7.35(\mathrm{~d}, 1 \mathrm{H}, 6-\mathrm{H}$, $\left.\mathrm{J}_{6,5}=8.8\right), 7.91\left(\mathrm{~d}, 1 \mathrm{H}, 5-\mathrm{H}, \mathrm{J}_{5,6}=8.8\right)$.

MS (m/z, \%): $271(\mathrm{M}+, 20), 256$ (- Me, 25), 242 (- HCO, 7), 214 (- HCO, - CO, 10).

Anal. calc. for $\mathrm{C}_{15} \mathrm{H}_{13} \mathrm{NO}_{4}$ : C 66.41, H 4.83, N 5.16; found: C 66.52, H 4.70, N 5.00.

\section{8-Formyl-4-methyldihydrofuro[2,3-h]coumarin-9-one (7g).}

Reaction mixture of the compound 9 synthesis was poured into water $(700 \mathrm{ml})$ and heated then for $5-7 \mathrm{~min}$ at $80-90^{\circ} \mathrm{C}$. The solution was left for a night, the formed precipitate was then filtered off and recrystallized from acetone.

Compound $7 \mathrm{~g}$, yield $=50 \%$, yellow crystals, mp $275^{\circ} \mathrm{C}$ (decomp.). $1 \mathrm{H} \mathrm{NMR}\left(\mathrm{CDCl}_{3}, \delta\right.$, ppm; J, $\mathrm{Hz}): 2.50\left(\mathrm{~d}, 3 \mathrm{H}, 4-\mathrm{Me}, \mathrm{J}_{\mathrm{Me}, 3}=1.2\right), 6.33\left(\mathrm{q}, 1 \mathrm{H}, 3-\mathrm{H}, \mathrm{J}_{3, \mathrm{Me}}=1.2\right), 7.17(\mathrm{~s}, 1 \mathrm{H}, 8-\mathrm{H}), 7.38(\mathrm{~d}, 1 \mathrm{H}$, $\left.6-\mathrm{H}_{,} \mathrm{J}_{6,5}=8.9\right), 7.75\left(\mathrm{~d}, 1 \mathrm{H}, 5-\mathrm{H}, \mathrm{J}_{5,6}=8.9\right), 9.88(\mathrm{~s}, 1 \mathrm{H},-\mathrm{COH})$.

MS (m/z, \%): 244 (M+, 100), 216 (- CO, 65), 215 (- HCO, 34), 187 (- CO, - HCO, 17), 159 ($\mathrm{HCO},-2 \mathrm{CO}, 16)$.

Anal. calc. for $\mathrm{C}_{13} \mathrm{H}_{8} \mathrm{O}_{5}$ : C 63.94, $\mathrm{H} 3.30$; found: $\mathrm{C} 63.84$, H 3.36

\section{8-Formyl-4-methyldihydrofuro[2,3-h]coumarin-9-one, 2,4-dinitrophenyl-hydrazone} (11).

Solution of 2,4-dinitrophenylhydrazine $(0.49 \mathrm{~g}, 0.002 \mathrm{~mol})$ in glacial acetic acid $(30 \mathrm{ml})$ was added to suspension of compound $7 \mathrm{~g}$ in glacial acetic acid $(50 \mathrm{ml})$. Reaction mixture was then stirred for $1 \mathrm{hr}$ at $20^{\circ} \mathrm{C}$ and $1 \mathrm{hr}$ at $60-80^{\circ} \mathrm{C}$. The formed precipitate was filtered off and recrystallized from acetone.

Compound 11, yield $=95 \%$, dark red crystals, mp 261-262 ${ }^{\circ} \mathrm{C}$. $1 \mathrm{H}$ NMR (acetone- $\mathrm{d}_{6}, \delta$, ppm; J, $\mathrm{Hz}$ ): 2.50 (d, 3H, 4-Me, J Me,3 =1.2), 6.39 (q, 1H, 3-H, J 3,Me $=1.2$ ), 7.59 (d, 1H, 6-H, J 6,5 =8.9), 
$7.78\left(\mathrm{~d}, 1 \mathrm{H}, 5-\mathrm{H}, \mathrm{J}_{5,6}=8.9\right), 7.98\left(\mathrm{~d}, 1 \mathrm{H}, 6^{\prime}-\mathrm{H}_{,} \mathrm{J}_{6,5}, 5^{\prime}=9.6\right), 8.43$ (dd, 1H, 5'-H, J $5^{\prime}, 6^{\prime}=9.6, \mathrm{~J}_{5}, 3^{\prime}$ =2.6), $8.84(\mathrm{~s}, 1 \mathrm{H},-\mathrm{CH}=), 8.88\left(\mathrm{~d}, 1 \mathrm{H}, 3^{\prime}-\mathrm{H}, \mathrm{J}_{3}{ }^{\prime}, 5,=2.6\right), 10.6(\mathrm{~s}, 1 \mathrm{H}, \mathrm{OH}), 11.74(\mathrm{~s}, 1 \mathrm{H}, \mathrm{NH})$.

MS (m/z, \%): $424(\mathrm{M}+, 27), 202\left(-\mathrm{CH}-\mathrm{CH}=\mathrm{N}-\mathrm{NH}-\mathrm{C}_{6} \mathrm{H}_{3}\left(\mathrm{NO}_{2}\right)_{2}, 40\right), 174$ (- CH-CH=N-NH$\left.\mathrm{C}_{6} \mathrm{H}_{3}\left(\mathrm{NO}_{2}\right)_{2},-\mathrm{CO}, 100\right), 215\left(-\mathrm{CH}=\mathrm{N}-\mathrm{NH}-\mathrm{C}_{6} \mathrm{H}_{3}\left(\mathrm{NO}_{2}\right)_{2}, 13\right), 171\left(-\mathrm{CH}=\mathrm{N}-\mathrm{NH}-\mathrm{C}_{6} \mathrm{H}_{3}\left(\mathrm{NO}_{2}\right)_{2}\right.$,$\left.\mathrm{CO}_{2}, 33\right)$.

Anal. calc. for $\mathrm{C}_{19} \mathrm{H}_{12} \mathrm{~N}_{4} \mathrm{O}_{8}$ : C 53.78, H 2.85, N 13.20; found: C 53.69, H 2.91, N 13.21.

Croton condensation of $\mathbf{2 b}$ with aldehydes. Synthesis of 8-(R-benzylideno)-4methyldihydrofuro[2,3-h]coumarin-9-ones (12).

Compound 2b $(0.5 \mathrm{~g}, 0.0023 \mathrm{~mol})$, glacial acetic acid $(12 \mathrm{ml})$, conc. $\mathrm{HCl}(6 \mathrm{ml})$ and Rbenzaldehyde $(0.019 \mathrm{~mol})$ were heated for $1 \mathrm{hr}$ under reflux. Precipitate was filtered off and recrystallized from DMSO.

\section{8-(4'-Bromobenzylideno)-4-methyldihydrofuro[2,3-h]coumarin-9-one(12b).}

yield $=85 \%$, yellow crystals, mp 335-336 ${ }^{\circ} \mathrm{C} .1 \mathrm{H} \mathrm{NMR}\left(\mathrm{DMSO}_{6} \mathrm{~d}_{6}, \delta\right.$, ppm; J, Hz): 2.47 (d, 3H, 4-Me, $\left.\mathrm{J}_{\mathrm{Me}, 3}=1.2\right), 6.36\left(\mathrm{q}, 1 \mathrm{H}, 3-\mathrm{H}, \mathrm{J}_{3, \mathrm{Me}}=1.2\right), 6.96(\mathrm{~s}, 1 \mathrm{H},=(\mathrm{Ph}) \mathrm{C}-\mathrm{H}), 7.48\left(\mathrm{~d}, 1 \mathrm{H}, 6-\mathrm{H}, \mathrm{J}_{6,5}\right.$ $=9.0), 7.70\left(\mathrm{~d}, 2 \mathrm{H}, 2^{\prime}-\mathrm{H}, \mathrm{J}_{2}{ }^{\prime}, 3^{\prime}=8.5\right), 7.92\left(\mathrm{~d}, 2 \mathrm{H}, 3^{\prime}-\mathrm{H}_{,} \mathrm{J}_{3^{\prime}, 2^{\prime}}=8.5\right), 8.16\left(\mathrm{~d}, 1 \mathrm{H}, 5-\mathrm{H}, \mathrm{J}_{5,6}=9.0\right)$. MS (m/z, \%): 382/384, (1Br), (M+, 79), 303 (- Br, 100), 353/355, (1Br), (- HCO, 11), 275 (- Br, - CO, 18).

Anal. calc. for $\mathrm{C}_{19} \mathrm{H}_{11} \mathrm{BrO}_{4}$ : C 59.55, H 2.89; Br 20.85; found: C 59.40, H 2.99, Br 20.69.

8-(4'-N,N-Dimethylaminobenzylideno)-4-methyldihydrofuro[2,3-h]coumarin-9-one (12c). yield $=87 \%$, yellow crystals, mp 315-317 ${ }^{\circ} \mathrm{C} .1 \mathrm{H}$ NMR $\left(\mathrm{DMSO}_{-} \mathrm{d}_{6}, \delta\right.$, ppm; J, Hz): 2.47 (d, 3H, 4Me, J Me,3 =0.9), 3.04 (s, 6H, N(Me)2), 6.33 (q, 1H, 3-H, J 3,Me =0.9), 6.82 (d, 2H, 3'-H, J'2' $=9.0), 6.91(\mathrm{~s}, 1 \mathrm{H},=(\mathrm{Ph}) \mathrm{C}-\mathrm{H}), 7.44\left(\mathrm{~d}, 1 \mathrm{H}, 6-\mathrm{H}, \mathrm{J}_{6,5}=8.7\right), 7.85\left(\mathrm{~d}, 2 \mathrm{H}, 2^{\prime}-\mathrm{H}, \mathrm{J}_{2}{ }^{\prime}, 3^{\prime}=9.0\right), 8.08$ $\left(\mathrm{d}, 1 \mathrm{H}, 5-\mathrm{H}, \mathrm{J}_{5,6}=8.7\right)$.

MS (m/z, \%): 347 (M+, 100), 346(- H, 22), 319 (- CO, 2), 290 (- CO, - HCO, 22).

Anal. calc. for $\mathrm{C}_{21} \mathrm{H}_{17} \mathrm{NO}_{4}$ : C 72.61, $\mathrm{H}$ 4.93, N 4.03; found: C 72.50, H 4.72, N 4.21. 


\section{8-(4'-Hydroxy-3'-methoxybenzylideno)-4-methyldihydrofuro[2,3-h]coumarin-9-one (12d).}

yield $=85 \%$, yellow crystals, mp 330-331 ${ }^{\circ} \mathrm{C} .1 \mathrm{H} \mathrm{NMR}\left(\mathrm{DMSO}_{6}, \delta, \mathrm{ppm} ; \mathrm{J}, \mathrm{Hz}\right): 2.44(\mathrm{~d}, 3 \mathrm{H}$, 4-Me, J Me,3 =0.9), 3.86 (s, 3H, OMe), 6.35 (q, 1H, 3-H, J 3,Me =0.9), 6.85 (d, 1H, 5' -H, J 5',6' =8.5) $6.90(\mathrm{~s}, 1 \mathrm{H},=(\mathrm{Ph}) \mathrm{C}-\mathrm{H}), 7.48\left(\mathrm{~d}, 1 \mathrm{H}, 6-\mathrm{H}, \mathrm{J}_{6,5}=9.0\right), 7.49\left(\mathrm{dd}, 1 \mathrm{H}, 6{ }^{\prime}-\mathrm{H}, \mathrm{J}_{6}{ }^{\prime}, 5,=8.5, \mathrm{~J}_{6}, 2^{\prime}\right.$, $=0.9), 7.54\left(\mathrm{~d}, 1 \mathrm{H}, 2^{\prime}-\mathrm{H}_{1} \mathrm{~J}_{2}{ }^{\prime}, 6^{\prime}=0.9\right), 8.09\left(\mathrm{~d}, 1 \mathrm{H}, 5-\mathrm{H}, \mathrm{J}_{5,6}=9.0\right), 9.94(\mathrm{~s}, 1 \mathrm{H}, \mathrm{OH})$.

MS (m/z, \%): $350(\mathrm{M}+, 100), 333(-\mathrm{OH}, 16), 319$ (- OMe, 14).

Anal. calc. for $\mathrm{C}_{20} \mathrm{H}_{14} \mathrm{NO}_{6}$ : C 68.57, $\mathrm{H} 4.03$; found: C 68.50, H 4.02.

\section{8-(2'-Nitrobenzylideno)-4-methyldihydrofuro[2,3-h]coumarin-9-one (12e).}

yield $=86 \%$, yellow crystals, mp 312-313 ${ }^{\circ} \mathrm{C} .1 \mathrm{H} \mathrm{NMR}\left(\mathrm{DMSO}_{6} \mathrm{~d}_{6}, \delta\right.$, ppm; J, Hz): 2.50 (d, 3H, 4Me, $\left.\mathrm{J}_{\mathrm{Me}, 3}=0.9\right), 6.38\left(\mathrm{q}, 1 \mathrm{H}, 3-\mathrm{H}, \mathrm{J}_{3, \mathrm{Me}}=0.9\right), 7.21(\mathrm{~s}, 1 \mathrm{H},=(\mathrm{Ph}) \mathrm{C}-\mathrm{H}), 7.41\left(\mathrm{~d}, 1 \mathrm{H}, 6-\mathrm{H}, \mathrm{J}_{6,5}\right.$ =8.0), $7.70\left(\mathrm{~m}, 1 \mathrm{H}, 4^{\prime}-\mathrm{H}\right), 7.87$ (m, 1H, 5'-H), 8.11-8.23 (m, 3H, 3'-H, 6'-H, 5-H).

MS (m/z, \%): 349 (M+, 17), 332(- OH, 17), 319 (- NO, 100), $303\left(-\mathrm{NO}_{2}, 98\right), 275$ (- $\mathrm{NO}_{2},-\mathrm{CO}$, 98).

Anal. calc. for $\mathrm{C}_{19} \mathrm{H}_{11} \mathrm{NO}_{6}$ : C 65.33, H 3.17, $\mathrm{N}$ 4.01; found: $\mathrm{C} 65.21, \mathrm{H} 3.03, \mathrm{~N} 4.17$.

\section{8-(2'-Hydroxybenzylideno)-4-methyldihydrofuro[2,3-h]coumarin-9-one(12f).}

yield $=87 \%$, yellow crystals, mp 296-297 C. 1H NMR (DMSO-d 6 , $\delta$, ppm; J, Hz): 2.47 (d, 3H, 4-Me, $\left.\mathrm{J}_{\mathrm{Me}, 3}=0.9\right), 6.35$ (q, 1H, 3-H, J $\left.3, \mathrm{Me}=0.9\right), 6.95$ (s, 1H, =(Ph)C-H), 6.97 (d, 1H, 3'-H, J 3',4' =8.0), 7.29 (m, 1H, 4'-H, 5'-H), $7.46\left(\mathrm{~d}, 1 \mathrm{H}, 6-\mathrm{H}^{\prime} \mathrm{J}_{6,5}=9.0\right), 8.11$ (d, 1H, 6’-H, J $6^{\prime}, 5$, =8.0), 8.12 $\left(\mathrm{d}, 1 \mathrm{H}, 5-\mathrm{H}, \mathrm{J}_{5,6}=9.0\right), 10.21(\mathrm{~s}, 1 \mathrm{H}, \mathrm{OH})$.

MS (m/z, \%): $320(\mathrm{M}+, 81), 303(-\mathrm{OH}, 23), 202$ (-OH, - C=C-Ph, 100), 174 (- OH, - C=C-Ph, $\mathrm{CO}, 93)$.

Anal. calc. for $\mathrm{C}_{19} \mathrm{H}_{12} \mathrm{O}_{5}$ : C 71.24, H 3.78; found: C 71.10, H 3.60. 


\section{8-(4'-Nitrobenzylideno)-4-methyldihydrofuro[2,3-h]coumarin-9-one (12g).}

yield $=85 \%$, yellow crystals, mp 354-355 ${ }^{\circ} \mathrm{C} .1 \mathrm{H}$ NMR (DMSO-d 6 , $\delta$, ppm; J, Hz): 2.50 (d, 3H, 4Me, $\left.\mathrm{J}_{\mathrm{Me}, 3}=0.9\right), 6.38\left(\mathrm{q}, 1 \mathrm{H}, 3-\mathrm{H}, \mathrm{J}_{3, \mathrm{Me}}=0.9\right), 7.08(\mathrm{~s}, 1 \mathrm{H},=(\mathrm{Ph}) \mathrm{C}-\mathrm{H}), 7.50$ (d, 1H, 6-H, J 6,5 $=9.0), 8.18\left(\mathrm{~d}, 2 \mathrm{H}, 2^{\prime}-\mathrm{H}_{1} \mathrm{~J}_{2}{ }^{\prime}, 3^{\prime}=8.5\right), 8.23\left(\mathrm{~d}, 1 \mathrm{H}, 5-\mathrm{H}, \mathrm{J}_{5,6}=9.0\right) .8 .30$ (d, 2H, 3'-H, J $\left.3^{\prime} 2^{\prime}=8.5\right)$ MS (m/z, \%): $349(\mathrm{M}+, 100), 332(-\mathrm{OH}, 16), 303\left(-\mathrm{NO}_{2}, 20\right), 302\left(-\mathrm{HNO}_{2}, 57\right), 274\left(-\mathrm{HNO}_{2}\right.$, $\mathrm{CO}, 98)$.

Anal. calc. for $\mathrm{C}_{19} \mathrm{H}_{11} \mathrm{NO}_{6}$ : C 65.33, H 3.17, N 4.01; found: C 65.21, H 3.03, N 4.17.

\section{8-(4', $\alpha$-Dimethylbenzylideno)-4-methyldihydrofuro[2,3-h]coumarin-9-one(12h).}

yield $=86 \%$, yellow crystals, mp 263-264 ${ }^{\circ} \mathrm{C} .1 \mathrm{H} \mathrm{NMR}\left(\mathrm{DMSO}_{6}, \delta\right.$, ppm; J, Hz): 2.40 (s, 3H, pMe), 2.50 (d, 3H, 4-Me, J Me,3 =1.0), 2.69 (s, 3H, =(Ph)C-Me), 6.26 (q, 1H, 3-H, J 3,Me $=1.0$ ), 7.20 $\left(\mathrm{d}, 1 \mathrm{H}, 6-\mathrm{H}, \mathrm{J}_{6,5}=8.5\right), 7.65$ (d, 2H, 2'-H, J $\left.2^{\prime}, 3^{\prime}=8.4\right), 7.30\left(\mathrm{~d}, 2 \mathrm{H}, 3^{\prime}-\mathrm{H}_{,} \mathrm{J}_{3^{\prime}, 2^{\prime}}=8.5\right), 8.06(\mathrm{~d}, 1 \mathrm{H}$, $\left.5-\mathrm{H} \mathrm{J}_{5,6}=8.5\right)$.

MS (m/z, \%): 332, (M+, 86), $331(-\mathrm{H}, 90), 317,(-\mathrm{Me}, 100), 289$ (- Me, - CO, 7).

Anal. calc. for $\mathrm{C}_{21} \mathrm{H}_{16} \mathrm{O}_{4}$ : C 75.89, $\mathrm{H}$ 4.85; found: C 75.80, H 4.99.

\section{Synthesis of 8-(R-arylazo)-4-methyldihydrofuro[2,3-h]coumarin-9-ones 13.}

A mixture of compound 2b $(0.00093 \mathrm{~mol})$, glacial acetic acid $(10 \mathrm{ml})$, pyridine $(1 \mathrm{ml})$ and Rphenyldiazonium chloride was mixed for $8 \mathrm{hrs}$, washed then 3 times by acetone and recrystallized from DMCO.

4-Methyl-8-(4'-methylphenylazo)-4-methyldihydrofuro[2,3-h]coumarin-9-one13e, yield= 80\%, red crystals, mp 296-298 ${ }^{\circ} \mathrm{C}$. $1 \mathrm{H}$ NMR (DMSO-d 6 , $\delta$, ppm; J, Hz): 2.26 (s, 3H, 4'-Me), 2.47 (d,

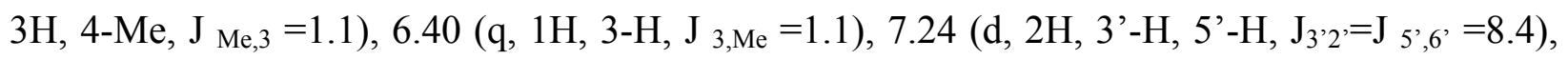
7.28 (d, 2H, 2'-H, 6'-H, J $\left.2^{\prime}, 3^{\prime}=\mathrm{J}_{6^{\prime}, 5^{\prime}}=8.4\right), 7.37$ (d, 6-H, J 6,5 $\left.=8.6\right), 8.11\left(\mathrm{~d}, 1 \mathrm{H}, 5-\mathrm{H}, \mathrm{J}_{5,6}=8.6\right)$, $11.01(\mathrm{~s}, 1 \mathrm{H}, \mathrm{NH})$.

MS (m/z, \%): $334(\mathrm{M}+, 100), 203\left(-4-\mathrm{MeC}_{6} \mathrm{H}_{4}-\mathrm{N}=\mathrm{N}-\mathrm{C}, 24\right), 174\left(-4-\mathrm{MeC}_{6} \mathrm{H}_{4}-\mathrm{N}=\mathrm{N}-\mathrm{C},-\mathrm{HCO}\right.$, 24).

Anal. calc. for $\mathrm{C}_{19} \mathrm{H}_{14} \mathrm{~N}_{2} \mathrm{O}_{4}$ : C 68.26, $\mathrm{H}$ 4.22, $\mathrm{N}$ 8.38; found: C 68.32, H 4.34, N 8.41. 
4-Methyl-8-(4'-chlorophenylazo)-4-methyldihydrofuro[2,3-h]coumarin-9-one (13f).

yield $=85 \%$, orange crystals, mp $320-322^{\circ} \mathrm{C} .1 \mathrm{H}$ NMR $\left(\mathrm{DMSO}_{6}, \delta\right.$, ppm; J, Hz): $2.48(\mathrm{~d}, 3 \mathrm{H}$, 4-Me, J Me,3 =1.1), 6.42 (q, 1H, 3-H, J 3,Me =1.1), 7.36 (m, 4H, 2'-H, 3'-H, 5'-H, 6'-H), 7.38 (d, $\left.1 \mathrm{H}, 6-\mathrm{H}, \mathrm{J}_{6,5}=8.6\right), 8.14\left(\mathrm{~d}, 1 \mathrm{H}, 5-\mathrm{H}, \mathrm{J}_{5,6}=8.6\right), 11.15(\mathrm{~s}, 1 \mathrm{H}, \mathrm{NH})$.

MS (m/z, \%): 354/356 (1Cl) (M+, 100), $319(-\mathrm{Cl}, 5), 291(-\mathrm{Cl},-\mathrm{CO}, 5), 202\left(-4-\mathrm{ClC}_{6} \mathrm{H}_{4}-\mathrm{N}=\mathrm{N}-\mathrm{C}\right.$, 44), $174\left(-4-\mathrm{ClC}_{6} \mathrm{H}_{4}-\mathrm{N}=\mathrm{N}-\mathrm{C},-\mathrm{CO}, 66\right)$.

Anal. calc. for $\mathrm{C}_{18} \mathrm{H}_{11} \mathrm{ClN}_{2} \mathrm{O}_{4}$ : C 60.94, H 3.13, Cl 9.99, N 7.90; found: C 60.84, H 3.23, Cl9.89 N 7.80.

\section{4-Methyl-8-(2'-nitrophenylazo)-dihydrofuro[2,3-h]coumarin-9-one (13g).}

yield $=81 \%$, yellow crystals, mp $350^{\circ} \mathrm{C} .1 \mathrm{H} \mathrm{NMR}\left(\mathrm{DMSO}_{6}, \delta\right.$, ppm; J, Hz): 2.48 (d, 3H, 4Me, $\left.\mathrm{J}_{\mathrm{Me}, 3}=1.1\right), 6.44$ (q, 1H, 3-H, J 3,Me $\left.=1.1\right), 7.17$ (s, 1H, 4'-H), 7.56 (d, 6-H, J 6,5 =8.8), 7.81 (m, 1H, 5’-H), 7.92 (m, 1H, 6'-H), 8.22 (d, 1H, 5-H, J 5,6 =8.8), 11.03 (s, 1H, NH).

MS (m/z, \%): $365(\mathrm{M}+, 64), 203\left(-2-\mathrm{NO}_{2} \mathrm{C}_{6} \mathrm{H}_{4}-\mathrm{N}=\mathrm{N}-\mathrm{C}, 90\right), 202\left(-2-\mathrm{NO}_{2} \mathrm{C}_{6} \mathrm{H}_{4}-\mathrm{NH}-\mathrm{N}=\mathrm{C}, 63\right)$, $174\left(-2-\mathrm{NO}_{2} \mathrm{C}_{6} \mathrm{H}_{4}-\mathrm{N}=\mathrm{N}=\mathrm{C},-\mathrm{HCO}, 100\right), 174 \quad\left(-2-\mathrm{NO}_{2} \mathrm{C}_{6} \mathrm{H}_{4}-\mathrm{NH}-\mathrm{N}=\mathrm{C},-\mathrm{CO}, 100\right), 146 \quad(-2-$ $\left.\mathrm{NO}_{2} \mathrm{C}_{6} \mathrm{H}_{4}-\mathrm{NH}-\mathrm{N}=\mathrm{C},-2 \mathrm{CO}, 16\right), 145\left(-2-\mathrm{NO}_{2} \mathrm{C}_{6} \mathrm{H}_{4}-\mathrm{NH}-\mathrm{N}=\mathrm{C},-\mathrm{CO},-\mathrm{HCO}, 10\right)$.

Anal. calc. for $\mathrm{C}_{18} \mathrm{H}_{11} \mathrm{~N}_{3} \mathrm{O}_{6}$ : C 59.18, H 3.04, N 11.50; found: C 59.26, H 3.30, N 11.37.

\section{4-Methyl-8-(2'4'-dinitrophenylazo)-4-methyldihydrofuro[2,3-h]coumarin-9-one (13h).} yield $=80 \%$, yellow crystals, mp $350^{\circ} \mathrm{C} .1 \mathrm{H} \mathrm{NMR}\left(\mathrm{DMSO}_{6}, \delta, \mathrm{ppm} ; \mathrm{J}, \mathrm{Hz}\right): 2.50$ (d, 3H, 4-Me, $\left.\mathrm{J}_{\mathrm{Me}, 3}=1.1\right), 6.41$ (q, 1H, 3-H, $\left.\mathrm{J}_{3, \mathrm{Me}}=1.1\right), 7.56$ (d, 6-H, J 6,5 =8.8), 7.20 (d, 1H, 6'-H, J 6,5 =9.4), $8.31\left(\mathrm{~d}, 1 \mathrm{H}, 5-\mathrm{H}_{1} \mathrm{~J}_{5,6}=8.8\right), 8.61\left(\mathrm{dd}, 1 \mathrm{H}, 5^{\prime}-\mathrm{H}_{\text {, J }}{ }^{\prime}, 6^{\prime}=9.4, \mathrm{~J}_{5}^{\prime}, 3^{\prime}=2.6\right), 9.03$ (d, 1H, 3'-H, J $\left.{ }_{3}{ }_{5},=2.6\right), 11.38(\mathrm{~s}, 0.62 \mathrm{H}, \mathrm{NH}), 13.65(\mathrm{~s}, 0.38 \mathrm{H}, \mathrm{OH})$.

MS (m/z, \%): $410(\mathrm{M}+, 42), 202\left(-2-\mathrm{NO}_{2}-4-\mathrm{NO}_{2} \mathrm{C}_{6} \mathrm{H}_{3}, 70\right), 174\left(-2-\mathrm{NO}_{2}-4-\mathrm{NO}_{2} \mathrm{C}_{6} \mathrm{H}_{3},-\mathrm{CO}, 100\right)$. Anal. calc. for $\mathrm{C}_{18} \mathrm{H}_{10} \mathrm{~N}_{4} \mathrm{O}_{8}$ : C 52.69, H 2.46, N 13.66; found: C 52.50, H 2.41, N 13.66. 
4-Methyl-8-(4'-diethylaminophenylazo)-4-methyldihydrofuro[2,3-h]coumarin-9-one (13i). yield $=85 \%$, red crystals, mp $350^{\circ} \mathrm{C} .1 \mathrm{H} \mathrm{NMR}\left(\mathrm{DMSO}_{6}, \delta\right.$, ppm; J, Hz): 1.07 (t, 3H, Me, J Me,$\left.\mathrm{CH} 2-_{-}=7.5\right), 2.49\left(\mathrm{~d}, 3 \mathrm{H}, 4-\mathrm{Me}, \mathrm{J}_{\mathrm{Me}, 3}=1.1\right), 3.51$ (q, 2H, -CH2-, J-CH2-,Me $\left.=7.5\right), 6.41$ (q, 1H, 3-H, J 3,Me $=1.1), 7.38\left(\mathrm{~d}, 6-\mathrm{H}, \mathrm{J}_{6,5}=8.9\right), 7.51$ (m, 4H, 2’'-H, 3'-H, 5'-H, 6'-H), 8.14 (d, 1H, 5-H, J 5,6 $=8.9), 11.24(\mathrm{~s}, 1 \mathrm{H}, \mathrm{NH})$.

MS (m/z, \%): $391(\mathrm{M}+, 2)$.

Anal. calc. for $\mathrm{C}_{22} \mathrm{H}_{21} \mathrm{~N}_{3} \mathrm{O}_{4}$ : C 67.51, H 5.41, N 10.73; found: C 67.82, H 5.54, N 10.62.

4-Methyl-8-(2'-ethylphenylazo)-4-methyldihydrofuro[2,3-h]coumarin-9-one (13j). yield $=70 \%$, red crystals, mp 350 ${ }^{\circ} \mathrm{C}$. $1 \mathrm{H} \mathrm{NMR}\left(\mathrm{DMSO}_{6}, \delta\right.$, ppm; J, Hz): 1.40 (t, 3H, Me, J Me,$\left.\mathrm{CH} 2-_{-}=7.5\right), 2.50\left(\mathrm{~d}, 3 \mathrm{H}, 4-\mathrm{Me}, \mathrm{J}_{\mathrm{Me}, 3}=1.1\right), 2.90$ (q, 2H, $\left.-\mathrm{CH}_{2-}, \mathrm{J}_{-\mathrm{CH} 2-, \mathrm{Me}}=7.5\right), 6.41$ (q, 1H, 3-H, J 3,Me $=1.1), 7.52$ (m, 4H, 3'-H, 4'-H, 5'-H, 6'-H), 7.22 (d, 6-H, J 6,5 =8.9), 8.02 (d, 1H, 5-H, J 5,6 $=8.9), 10.00(\mathrm{~s}, 0.6 \mathrm{H}, \mathrm{NH}), 12.20(\mathrm{~s}, 0.4 \mathrm{H}, \mathrm{OH})$.

$\operatorname{MS}(\mathrm{m} / \mathrm{z}, \%): 348(\mathrm{M}+, 100)$.

Anal. calc. for $\mathrm{C}_{20} \mathrm{H}_{16} \mathrm{~N}_{2} \mathrm{O}_{4}$ : C 68.96, $\mathrm{H}$ 4.63, N 8.04; found: $\mathrm{C} 68.76, \mathrm{H}$ 4.57, $\mathrm{N}$ 8.27.

4-Methyl-8-(3'-ethylphenylazo)-4-methyldihydrofuro[2,3-h]coumarin-9-one (13k). yield $=70 \%$, red crystals, mp 350 ${ }^{\circ} \mathrm{C}$. $1 \mathrm{H} \mathrm{NMR}\left(\mathrm{DMSO}_{6}, \delta\right.$, ppm; J, Hz): 1.25 (t, 3H, Me, J Me,$\mathrm{CH} 2-=7.5), 2.50\left(\mathrm{~d}, 3 \mathrm{H}, 4-\mathrm{Me}, \mathrm{J}_{\mathrm{Me}, 3}=1.1\right), 2.60$ (q, 2H, - $\left.\mathrm{CH}_{2-}, \mathrm{J}_{-\mathrm{CH} 2-\mathrm{Me}}=7.5\right), 6.31$ (q, 1H, 3-H, J 3,Me $=1.1), 7.22$ (m, 4H, 2'-H, 4'-H, 5'-H, 6'-H), 7.31 (d, 6-H, J 6,5 =8.9), 8.02 (d, 1H, 5-H, J 5,6 $=8.9), 10.90(\mathrm{~s}, 1 \mathrm{H}, \mathrm{NH})$.

MS (m/z, \%): $348(\mathrm{M}+, 100), 202\left(-3-\mathrm{C}_{2} \mathrm{H}_{5} \mathrm{C}_{6} \mathrm{H}_{4}-\mathrm{NH}-\mathrm{N}=\mathrm{CH}, 24\right)$.

Anal. calc. for $\mathrm{C}_{20} \mathrm{H}_{16} \mathrm{~N}_{2} \mathrm{O}_{4}$ : C 68.96, $\mathrm{H}$ 4.63, N 8.04; found: C 68.81, H 4.47, N 8.15.

4-Methyl-8-(4'-ethylphenylazo)-4-methyldihydrofuro[2,3-h]coumarin-9-one (13I). yield $=75 \%$, red crystals, mp 280-282 ${ }^{\circ} \mathrm{C} .1 \mathrm{H} \mathrm{NMR}\left(\mathrm{DMSO}_{6}, \delta\right.$, ppm; J, Hz): 1.2 (t, 3H, Me, J Me,-CH2- $=7.5), 2.40\left(\mathrm{~d}, 3 \mathrm{H}, 4-\mathrm{Me}, \mathrm{J}_{\mathrm{Me}, 3}=1.1\right), 2.60$ (q, 2H, $\left.-\mathrm{CH}_{2^{-}}, \mathrm{J}_{-\mathrm{CH} 2-\mathrm{Me}}=7.5\right), 6.40$ (q, 1H, 3-

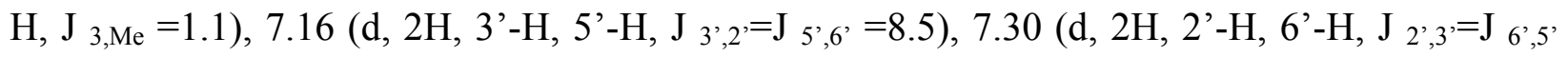
$=8.5), 7.36\left(\mathrm{~d}, 6-\mathrm{H}_{1} \mathrm{~J}_{6,5}=8.9\right), 8.10\left(\mathrm{~d}, 1 \mathrm{H}, 5-\mathrm{H}, \mathrm{J}_{5,6}=8.9\right), 11.02(\mathrm{~s}, 1 \mathrm{H}, \mathrm{NH})$. 
MS (m/z, \%): $348(\mathrm{M}+, 64), 203\left(-4-\mathrm{C}_{2} \mathrm{H}_{5} \mathrm{C}_{6} \mathrm{H}_{4}-\mathrm{NH}-\mathrm{N}=\mathrm{CH}, 26\right), 202\left(-4-\mathrm{C}_{2} \mathrm{H}_{5} \mathrm{C}_{6} \mathrm{H}_{4}-\mathrm{NH}-\mathrm{N}=\mathrm{C}\right.$, 36), $174\left(-4-\mathrm{C}_{2} \mathrm{H}_{5} \mathrm{C}_{6} \mathrm{H}_{4}-\mathrm{NH}-\mathrm{N}=\mathrm{CH}, \mathrm{CO}, 77\right),, 146\left(-4-\mathrm{C}_{2} \mathrm{H}_{5} \mathrm{C}_{6} \mathrm{H}_{4}-\mathrm{NH}-\mathrm{N}=\mathrm{C},-2 \mathrm{CO}, 13\right)$.

Anal. calc. for $\mathrm{C}_{20} \mathrm{H}_{16} \mathrm{~N}_{2} \mathrm{O}_{4}$ : C 68.96, H 4.63, N 8.04; found: C 68.86, H 4.50, N 8.17.

4-Methyl-8-(2',3'-dimethylphenylazo)-4-methyldihydrofuro[2,3-h]coumarin-9-one (13m). yield $=70 \%$, red crystals, mp 278-280 ${ }^{\circ} \mathrm{C} .1 \mathrm{H}$ NMR $\left(\mathrm{DMSO}_{6}, \delta\right.$, ppm; J, Hz): 2.51 (d, 3H, 4-Me, $\mathrm{J}_{\mathrm{Me}, 3}=1.1$ ), 3.30 (s, 6H, 2'-Me, 3'-Me), 6.40 (q, 1H, 3-H, J 3,Me $=1.1$ ), 6.35-6.91 (m, 3H, 4'-H, 5'-H, 6'-H), 7.41 (d, 6-H, J 6,5 =8.8), 8.21 (d, 1H, 5-H, J 5,6 =8.8), 9.81 (s, 1H, NH).

MS (m/z, \%): $348(\mathrm{M}+, 100)$.

Anal. calc. for $\mathrm{C}_{20} \mathrm{H}_{16} \mathrm{~N}_{2} \mathrm{O}_{4}$ : C 68.96, H 4.63, N 8.04; found: C 68.81, H 4.70, N 8.27.

4-Methyl-8-(2'-methylphenylazo)-4-methyldihydrofuro[2,3-h]coumarin-9-one (13n). yield $=70 \%$, red crystals, mp $350^{\circ} \mathrm{C} .1 \mathrm{H}$ NMR $\left(\right.$ DMSO-d $_{6}, \delta$, ppm; J, Hz): 2.35 (s, 3H, 2'-Me), 2.50 (d, 3H, 4-Me, J Me,3 $=1.1$ ), 6.41 (q, 1H, 3-H, J $3, \mathrm{Me}=1.1$ ), 6.96-8.10, (m, 4H, 3'-H, 4'-H, 5'H, 6'-H), 7.42 (d, 6-H, J $6,5=8.9), 8.12$ (d, 1H, 5-H, J $5,6=8.9), 9.75(\mathrm{~s}, 1 \mathrm{H}, \mathrm{NH})$.

MS (m/z, \%): $334(\mathrm{M}+, 100)$.

Anal. calc. for $\mathrm{C}_{19} \mathrm{H}_{14} \mathrm{~N}_{2} \mathrm{O}_{4}$ : C 68.26, H 4.22, N 8.38; found: C 6.98, H 4.09, N 8.43.

4-Methyl-8-(4'-bromophenylazo)-4-methyldihydrofuro[2,3-h]coumarin-9-one (130). yield $=85 \%$, orange crystals, mp $350^{\circ} \mathrm{C} .1 \mathrm{H}$ NMR $\left(\right.$ DMSO- $_{6}, \delta$, ppm; J, Hz): 2.49 (d, 3H, 4-Me,

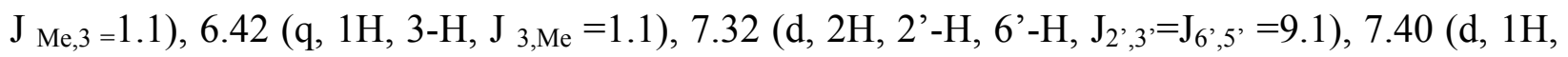

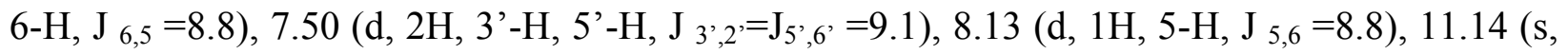
$1 \mathrm{H}, \mathrm{NH})$.

MS (m/z, \%): 398/400 (1Br) (M+, 100), $202\left(-4-\mathrm{BrC}_{6} \mathrm{H}_{4}-\mathrm{N}=\mathrm{N}-\mathrm{C}, 33\right), 174\left(-4-\mathrm{BrC}_{6} \mathrm{H}_{4}-\mathrm{N}=\mathrm{N}-\mathrm{C},-\right.$ $\mathrm{CO}, 77)$.

Anal. calc. for $\mathrm{C}_{18} \mathrm{H}_{11} \mathrm{BrN}_{2} \mathrm{O}_{4}$ : C 54.16, H 2.78, $\mathrm{Br} 20.02, \mathrm{~N}$ 7.02; found: C 54.25, H 2.67, Br 20.34 N 7.34 . 
4-Methyl-8-(3'-nitrophenylazo)-4-methyldihydrofuro[2,3-h]coumarin-9-one (13p), yield $=81 \%$, yellow crystals, mp $350^{\circ} \mathrm{C} .1 \mathrm{H} \mathrm{NMR}\left(\mathrm{DMSO}_{6}, \delta, \mathrm{ppm} ; \mathrm{J}, \mathrm{Hz}\right): 2.48(\mathrm{~d}, 3 \mathrm{H}, 4-\mathrm{Me}$, $\left.\mathrm{J}_{\mathrm{Me}, 3}=1.1\right), 6.42\left(\mathrm{q}, 1 \mathrm{H}, 3-\mathrm{H}, \mathrm{J}_{3, \mathrm{Me}}=1.1\right), 7.40$ (d, 6-H, J 6,5 =8.9), 7.61 (m, 1H, 5'-H), 7.22 (d, $\left.1 \mathrm{H}, 6^{\prime}-\mathrm{H}, \mathrm{J}_{6}, 5^{\prime}=8.0\right), 7.76\left(\mathrm{~d}, 1 \mathrm{H}, 4^{\prime}-\mathrm{H}, \mathrm{J}_{4}, 5^{\prime}=8.0\right), 8.12$ (s, 1H, 2'-H), 8.17 (d, 1H, 5-H, J $\left.{ }_{5,6}=8.9\right), 11.30(\mathrm{~s}, 1 \mathrm{H}, \mathrm{NH})$.

MS (m/z, \%): $365(\mathrm{M}+, 100), 202\left(-3-\mathrm{NO}_{2} \mathrm{C}_{6} \mathrm{H}_{4}-\mathrm{NH}-\mathrm{N}=\mathrm{C}, 63\right), 174\left(-3-\mathrm{NO}_{2} \mathrm{C}_{6} \mathrm{H}_{4}-\mathrm{NH}-\mathrm{N}=\mathrm{C},-\right.$ $\mathrm{CO}, 94)$.

Anal. calc. for $\mathrm{C}_{18} \mathrm{H}_{11} \mathrm{~N}_{3} \mathrm{O}_{6}$ : C 59.18, H 3.04, N 11.50; found: C 59.28, H 3.24, N 11.30.

Synthesis of 4-methyldihydrofuro[2,3-h]coumarin-8,9-diones, 8-(N-acetyl-N-aryl) hydrazones 14.

A solution of compound $13(0.00026 \mathrm{~mol})$ in acetic anhydride $(6 \mathrm{ml})$ and pyridine $(6 \mathrm{ml})$ was stirred for $1 \mathrm{hr}$ at $50^{\circ} \mathrm{C}$ and poured then into water $(50 \mathrm{ml})$. The product was filtered off and recrystallized from acetic anhydride.

\section{4-Methyldihydrofuro[2,3-h] coumarin-8,9-dione,8- $N$-acetyl- $N-\left(4^{\prime}\right.$-chlorophenyl) hydrazone} 14d,

yield $=75 \%$, yellow crystals, mp $320-322^{\circ} \mathrm{C} .1 \mathrm{H} \mathrm{NMR}\left(\mathrm{DMSO}_{6}, \delta\right.$, ppm; J, Hz): $2.41(\mathrm{~d}, 3 \mathrm{H}$, 4-Me, J Me,3 =1.1), 2.63 (s, 3H, Ac), 6.27 (q, 1H, 3-H, J 3,Me =1.1), 7.22 (dd, 2H, 2'-H, 6'-H, J $\left.2^{\prime}, 3^{\prime}=\mathrm{J}_{6^{\prime}, 5^{\prime}}=8.8, \mathrm{~J}_{2^{\prime}, 6^{\prime}}=2.1\right), 7.45\left(\mathrm{dd}, 2 \mathrm{H}, 3^{\prime}-\mathrm{H}, 5^{\prime}-\mathrm{H}, \mathrm{J}_{3^{\prime}, 2^{\prime}}=\mathrm{J}_{5^{\prime}, 6^{\prime}}=8.8, \mathrm{~J}_{3^{\prime}, 5^{\prime}}=2.1\right), 6.66(\mathrm{~d}, 1 \mathrm{H}, 6-$ $\left.\mathrm{H}, \mathrm{J}_{6,5}=8.4\right), 7.77\left(\mathrm{~d}, 1 \mathrm{H}, 5-\mathrm{H}, \mathrm{J}_{5,6}=8.4\right)$.

MS (m/z, \%): 396/398 (1Cl) (M+, 7), 354/356 (1Cl), $\left(-\mathrm{CH}_{2}=\mathrm{C}=\mathrm{O}, 100\right), 203\left(-\mathrm{CH}_{2}=\mathrm{C}=\mathrm{O},-4-\right.$ $\left.\mathrm{ClC}_{6} \mathrm{H}_{4}-\mathrm{N}=\mathrm{N}-\mathrm{C}, 31\right), 202\left(-\mathrm{CH}_{2}=\mathrm{C}=\mathrm{O},-4-\mathrm{ClC}_{6} \mathrm{H}_{4}-\mathrm{NH}-\mathrm{N}=\mathrm{CH}, 24\right), 174\left(-\mathrm{CH}_{2}=\mathrm{C}=\mathrm{O},-4-\mathrm{ClC}_{6} \mathrm{H}_{4}-\right.$ $\mathrm{NH}-\mathrm{N}=\mathrm{C},-\mathrm{CO}, 31), 174\left(-\mathrm{CH}_{2}=\mathrm{C}=\mathrm{O},-4-\mathrm{ClC}_{6} \mathrm{H}_{4}-\mathrm{N}=\mathrm{N}-\mathrm{C},-\mathrm{HCO}, 31\right)$

Anal. calc. for $\mathrm{C}_{20} \mathrm{H}_{13} \mathrm{ClN}_{2} \mathrm{O}_{5}$ : C 60.54, H 3.30, Cl 8.93, N 7.06; found: C 60.44, H 3.41, Cl 8.89 N 6.83 
4-Methyldihydrofuro[2,3-h]coumarin-8,9-dione,8- $N$-acetyl- $N$-(4'-methylphenyl) hydrazone (14e).

yield $=80 \%$, yellow crystals, mp 286-288 ${ }^{\circ} \mathrm{C} .1 \mathrm{H} \mathrm{NMR}\left(\mathrm{DMSO}-\mathrm{d}_{6}, \delta\right.$, ppm; J, Hz): 2.40 (s, 3H, 4'Me), 2.41 (d, 3H, 4-Me, J Me,3 =1.1), 2.42 (s, 3H, NAc), 2.63 (s, 3H, Ac), 6.38 (q, 1H, 3-H, J 3,Me =1.1), 7.24 (m, 4H, 2'-H, 3'-H, 5'-H, 6'-H,), 6.72 (d, 1H, 6-H, J 6,5 =8.6), 8.01 (d, 1H, 5-H, J 5,6 $=8.6$ ).

MS (m/z, \%): $376(\mathrm{M}+, 2), 334\left(-\mathrm{CH}_{2}=\mathrm{C}=\mathrm{O}, 100\right)$.

Anal. calc. for $\mathrm{C}_{21} \mathrm{H}_{16} \mathrm{~N}_{2} \mathrm{O}_{5}$ : C 67.02, $\mathrm{H}$ 4.28, N 7.44; found: C 66.85, H 4.02, N 7.52.

\section{Synthesis of 6-(R-phenylazo)-4-methyldihydrofuro[2,3-h]coumarin-9-ones (15 a-e).}

Compound $2 \mathrm{~b}(0.00046 \mathrm{~mol})$ was dissolved in $3 \%$-solution of $\mathrm{NaOH}$ at $80^{\circ} \mathrm{C}$ and cooled then till room temperature. R-Phenyldiazonium tetrafluoroborate $(0.00046 \mathrm{~mol})$ was then added to the prepared solution of $2 \mathrm{~b}$. After stirring for $30 \mathrm{~min}$ reaction mixture was acidified. The precipitate was filtered off and recrystallized from chloroform.

6-(3'-Ethylphenylazo)-4-methyldihydrofuro[2,3-h]coumarin-9-one 15d, yield $=70 \%$, yellow crystals, mp $350^{\circ} \mathrm{C} .1 \mathrm{H} \mathrm{NMR}\left(\mathrm{DMSO}_{6}, \delta, \mathrm{ppm} ; \mathrm{J}, \mathrm{Hz}\right): 1.33$ (t, 3H, Me, J Me,-CH2- $=7.5), 2.51\left(\mathrm{~d}, 3 \mathrm{H}, 4-\mathrm{Me}, \mathrm{J}_{\mathrm{Me}, 3}=1.1\right), 2.78\left(\mathrm{q}, 2 \mathrm{H},-\mathrm{CH}_{2^{-}}, \mathrm{J}_{-\mathrm{CH} 2-\mathrm{Me}}=7.5\right), 6.31$ (q, 1H, 3$\mathrm{H}, \mathrm{J}_{3, \mathrm{Me}}=1.1$ ), 7.53 (m, 4H, 2'-H, 4'-H, 5'-H, 6'-H), 8.25 (c, 1H, 5-H).

MS (m/z, \%): $348(\mathrm{M}+, 100), 215\left(-3-\mathrm{C}_{2} \mathrm{H}_{5} \mathrm{C}_{6} \mathrm{H}_{4}-\mathrm{N}=\mathrm{N}, 40\right)$.

Anal. calc. for $\mathrm{C}_{20} \mathrm{H}_{16} \mathrm{~N}_{2} \mathrm{O}_{4}$ : C 68.96, $\mathrm{H}$ 4.63, N 8.04; found: C 68.81, H 4.47, $\mathrm{N}$ 8.15.

\section{4-Methyl-6-(4'-dimethylaminophenylazo)dihydrofuro[2,3-h]coumarin-9-one (15e).}

yield $=85 \%$, red crystals, mp $350^{\circ} \mathrm{C} .1 \mathrm{H} \mathrm{NMR}\left(\right.$ DMSO-d $_{6}, \delta$, ppm; J, Hz): 2.50 (d, 3H, 4-Me, J $\mathrm{Me}, 3=1.1), 3.13\left(\mathrm{~s}, 6 \mathrm{H}, \mathrm{Me}_{2} \mathrm{~N}\right), 4.89$ (s, 2H, $\mathrm{CH}_{2}$ ), 6.27 (q, 1H, 3-H, J3,Me $\left.=1.1\right), 6.75$ (d, 2H, 3'-H,

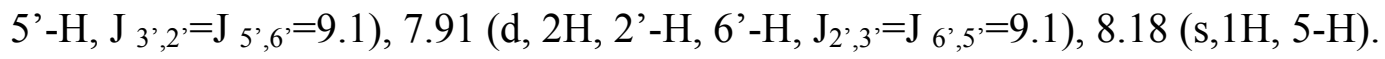

$\operatorname{MS}(\mathrm{m} / \mathrm{z}, \%): 363(\mathrm{M}+, 100)$.

Anal. calc. for $\mathrm{C}_{20} \mathrm{H}_{17} \mathrm{~N}_{3} \mathrm{O}_{4}$ : C 66.11, H 4.72, N 11.56; found: C 68.82, H 4.54, N 8.21. 


\section{6-Acetamido-4-methyldihydrofuro[2,3-h]coumarin-9-one (16b).}

6-Amino-4-methyldihydrofuro[2,3-h]coumarin-9-one $16 \mathrm{a}(0.0004 \mathrm{~mol})$ and acetic anhydride $(10 \mathrm{ml})$ were heated for $10 \mathrm{~min}$ and cooled then until a precipitate formed.

The product was recrystallized from acetic acid, white crystals, yield $=90 \%, \mathrm{mp} 279-281^{\circ} \mathrm{C} .1 \mathrm{H}$ NMR (DMSO-d 6 ,, ppm; J, Hz): 2.38 (d, 3H, 4-Me, J Me,3 =1.1), 2.13 (s, 3H, Ac), 4.98 (s, $\left.2 \mathrm{H}, \mathrm{CH}_{2}\right), 6.33$ (q, 1H, 3-H, J $\left.3, \mathrm{Me}=1.1\right), 8.52(\mathrm{~s}, 1 \mathrm{H}, 5-\mathrm{H}), 9.99$ (s, 1H, NH).

MS (m/z, \%): $273(\mathrm{M}+, 90), 231 \quad\left(-\mathrm{CH}_{2}=\mathrm{C}=\mathrm{O}, 100\right), 203 \quad\left(-\mathrm{CH}_{2}=\mathrm{C}=\mathrm{O},-\mathrm{CO}, 65\right), 175$ $\left.\mathrm{CH}_{2}=\mathrm{C}=\mathrm{O},-2 \mathrm{CO}, 47\right), 147\left(-\mathrm{CH}_{2}=\mathrm{C}=\mathrm{O},-3 \mathrm{CO}, 6\right), 146\left(-\mathrm{CH}_{2}=\mathrm{C}=\mathrm{O},-2 \mathrm{CO},-\mathrm{HCO}, 10\right), 118(-$ $\left.\mathrm{CH}_{2}=\mathrm{C}=\mathrm{O},-3 \mathrm{CO},-\mathrm{HCO}, 16\right)$.

Anal. calc. for $\mathrm{C}_{14} \mathrm{H}_{11} \mathrm{NO}_{5}$ : C 61.54, H 4.06, N 5.13; found: C 61.50, H 4.15, N 5.12.

\section{6-Formamido-4-methyldihydrofuro[2,3-h]coumarin-9-one (16c).}

6-Amino-4-methyldihydrofuro[2,3-h]coumarin-9-one $16 \mathrm{a}(0.0004 \mathrm{~mol})$ and formic acid (5 $\mathrm{ml})$ were heated for $10 \mathrm{~min}$ and cooled then until a precipitate formed. The product was recrystallized from acetic acid, white crystals, yield $=90 \%, \mathrm{mp} 279-281^{\circ} \mathrm{C} .1 \mathrm{H} \mathrm{NMR}\left(\mathrm{DMSO}-\mathrm{d}_{6}\right.$, $\delta$, ppm; J, Hz): 2.40 (d, 3H, 4-Me, J Me,3 =1.1), $5.01\left(\mathrm{~s}, 2 \mathrm{H}, \mathrm{CH}_{2}\right), 6.35\left(\mathrm{q}, 1 \mathrm{H}, 3-\mathrm{H}, \mathrm{J}_{3, \mathrm{Me}}=1.1\right)$, 8.38 (s, 1H, CH), 8.69 (s, 1H, 5-H), 10.32 (s, 1H, NH).

MS (m/z, \%): $259(\mathrm{M}+, 100), 231$ (- CO, 20), 203 (- 2CO, 23), 175 (- 3CO, 36).

Anal. calc. for $\mathrm{C}_{13} \mathrm{H}_{9} \mathrm{NO}_{5}$ : C 60.24, H 3.50, N 5.40; found: C 60.20, H 3.55, N 5.39.

\section{6-Azido-4-methyldihydrofuro[2,3-h]coumarin-9-one (16e).}

To the solution of 4-methyldihydrofuro[2,3-h]coumarin-9-one 6-diazonium chloride $16 \mathrm{~d}$ (0.0011 mol) was added sodium azide $(0.0015 \mathrm{~mol})$. The precipitate was filtered off, dried and recrystallized from DMFA, yellow crystals, yield $=40 \%, \mathrm{mp} 204^{\circ} \mathrm{C} .1 \mathrm{H} \mathrm{NMR}\left(\mathrm{DMSO}-\mathrm{d}_{6}, \delta\right.$, ppm; J, Hz): 2.41 (d, 3H, 4-Me, J $\mathrm{Me}, 3=1.1), 4.82\left(\mathrm{~s}, 2 \mathrm{H}_{,} \mathrm{CH}_{2}\right), 6.25$ (q, 1H, 3-H, J $\left.3, \mathrm{Me}=1.1\right), 7.37$ $(\mathrm{s}, 1 \mathrm{H}, 5-\mathrm{H})$.

MS (m/z, \%): $257(\mathrm{M}+, 15), 229(-\mathrm{CO}, 100)$.

Anal. calc. for $\mathrm{C}_{12} \mathrm{H}_{7} \mathrm{~N}_{3} \mathrm{O}_{4}$ : C 56.04, $\mathrm{H}$ 2.74, N 16.34; found: C 56.10, H 2.81, N 16.27. 


\section{6-Acetamido-9-acetoxy-4-methylfuro[2,3-h]coumarin (17a).}

6-Amino-4-methyldihydrofuro[2,3-h]coumarin-9-one 16a (0.0004 mol), acetic anhydride (10 ml) and pyridine $(5 \mathrm{ml})$ were heated for $20 \mathrm{~min}$ and cooled then until a precipitate formed. The product was recrystallized from acetic anhydride, white crystals, yield $=80 \%, \mathrm{mp} 228-230^{\circ} \mathrm{C} .1 \mathrm{H}$

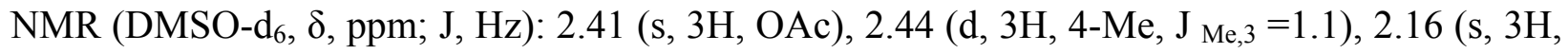
Ac), 6.39 (q, 1H, 3-H, J 3,Me $=1.1), 7.09$ (s, 1H, 5-H), 8.32 (s, 1H, 8-H), 10.16 (s, 1H, NH).

MS (m/z, \%): $315(\mathrm{M}+, 24), 273\left(-\mathrm{CH}_{2}=\mathrm{C}=\mathrm{O}, 100\right), 231\left(-2 \mathrm{CH}_{2}=\mathrm{C}=\mathrm{O}, 74\right), 203\left(-2 \mathrm{CH}_{2}=\mathrm{C}=\mathrm{O}\right.$, - $\mathrm{CO}, 16), 202\left(-2 \mathrm{CH}_{2}=\mathrm{C}=\mathrm{O},-\mathrm{HCO}, 13\right)$.

Anal. calc. for $\mathrm{C}_{16} \mathrm{H}_{13} \mathrm{NO}_{6}$ : C 60.95, $\mathrm{H}$ 4.16, N 4.44; found: $\mathrm{C} 60.72, \mathrm{H} 3.90, \mathrm{~N} 4.58$.

\section{6-Benzamido-9-benzoyloxy-4-methylfuro[2,3-h]coumarin (17b).}

6-Amino-4-methyldihydrofuro[2,3-h]coumarin-9-one $16 \mathrm{a}(0.0004 \mathrm{~mol})$, benzoyl chloride $(0.007$ mol), pyridine $(1 \mathrm{ml})$ and chloroform $(8 \mathrm{ml})$ were heated for $1 \mathrm{hr}$ and cooled then until a precipitate formed. The product was recrystallized from chloroform, white crystals, yield $=75 \%$,

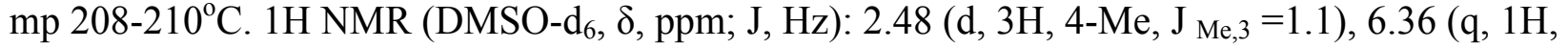
3-H, J 3 Me $=1.1), 7.57-8.30$ (m, 10H, H-Phenyls), 8.50 (s, 1H, 5-H), 7.91 (s, 1H, 8-H), 10.60 (s, $1 \mathrm{H}, \mathrm{NH})$.

MS (m/z, \%): $439(\mathrm{M}+, 100), 334$ (- PhCO, 20).

Anal. calc. for $\mathrm{C}_{26} \mathrm{H}_{17} \mathrm{NO}_{6}$ : C 60.95, H 4.16, N 4.44; found: C 60.72, H 3.90, N 4.58.

\section{9-Hydroxy-4-methyldihydrofuro[2,3-h]counarins 18 a-g.}

Substituted 4-methyldihydrofuro[2,3-h]coumarin-9-one (0.0014 mol) and NaBH4 (2 mol) were stirred in methanol $(20 \mathrm{ml})$ for $2 \mathrm{hrs}$ at room temperature and poured then into water $(30 \mathrm{ml})$. The precipitate was recrystallized from alcohol. 


\section{6-Chloro-9-hydroxy-4-methyldihydrofuro[2,3-h]coumarin (18a).}

light yellow crystals, yield=85\%, mp 235-237 C. $1 \mathrm{H} \mathrm{NMR} \mathrm{(DMSO-d} 6$, $\delta$, ppm; J, Hz): 2.47 (d, $\left.3 \mathrm{H}, 4-\mathrm{Me}, \mathrm{J}_{\mathrm{Me}, 3}=1.2\right), 4.63\left(\mathrm{dd}, 1 \mathrm{H}, 8 \mathrm{a}-\mathrm{H}, \mathrm{J}_{\text {hem }}=10.6, \mathrm{~J}_{8-\mathrm{a}, 9}=2.2\right), 4.82\left(\mathrm{dd}, 1 \mathrm{H}, 8 \mathrm{~b}-\mathrm{H}, \mathrm{J}_{\text {hem }}\right.$ $\left.=10.6, \mathrm{~J}_{8 \mathrm{~b}, 9}=6.2\right), 5.17\left(\mathrm{~d}, 1 \mathrm{H}, 9-\mathrm{OH}, \mathrm{J}_{9-\mathrm{OH}, 9}=6.0\right), 5.76\left(\mathrm{ddd}, 1 \mathrm{H}, 9-\mathrm{H}, \mathrm{J}_{9,8 \mathrm{~b}}=6.2, \mathrm{~J}_{9,9-\mathrm{OH}}=6.0\right.$, $\left.\mathrm{J}_{9,8 \mathrm{a}}=2.2\right), 6.19\left(\mathrm{q}, 1 \mathrm{H}, 3-\mathrm{H}, \mathrm{J}_{3, \mathrm{Me}}=1.2\right), 7.66(\mathrm{~s}, 1 \mathrm{H}, 5-\mathrm{H})$.

MS (m/z, \%): 252/254, (1 Cl), (M+, 100), 224/226, (1Cl), (- CO, 25), 195/197, (1 Cl), (- CO, H2O, 23), 237/239, (1Cl), (- Me, 10).

Anal. calc. for $\mathrm{C}_{12} \mathrm{H}_{9} \mathrm{ClO}_{4}$ : C 57.05, $\mathrm{H} 3.59, \mathrm{Cl} 14.03$; found: $\mathrm{C} 57.22, \mathrm{H} 3.72, \mathrm{Cl} 14.18$.

\section{6-Ethyl-9-hydroxy-4-methyldihydrofuro[2,3-h]coumarin (18b).}

light yellow crystals, yield=85\%, mp 205-206 ${ }^{\circ} \mathrm{C} .1 \mathrm{H} \mathrm{NMR}\left(\mathrm{DMSO}-\mathrm{d}_{6}, \delta, \mathrm{ppm} ; \mathrm{J}, \mathrm{Hz}\right): 1.23$ ( t, $\left.3 \mathrm{H}, \mathrm{Me}, \mathrm{J}_{\mathrm{Me}, \mathrm{CH} 2}=7.5\right), 2.44\left(\mathrm{~d}, 3 \mathrm{H}, 4-\mathrm{Me}, \mathrm{J}_{\mathrm{Me}, 3}=1.2\right), 4.56\left(\mathrm{dd}, 1 \mathrm{H}, 8 \mathrm{a}-\mathrm{H}, \mathrm{J}_{\text {hem }}=10.7, \mathrm{~J}_{8 \text {-a, } 9}\right.$ $=2.2), 4.72\left(\mathrm{dd}, 1 \mathrm{H}, 8 \mathrm{~b}-\mathrm{H}, \mathrm{J}_{\text {hem }}=10.7, \mathrm{~J}_{8 \mathrm{~b}, 9}=6.2\right), 4.98$ ( d, 1H, 9-OH, J 9-OH,9 =5.0), 5.68 (ddd, $\left.1 \mathrm{H}, 9-\mathrm{H}, \mathrm{J}_{9,8 \mathrm{~b}}=6.2, \mathrm{~J}_{9,9-\mathrm{OH}}=5.0, \mathrm{~J}_{9,8 \mathrm{a}}=2.2\right), 6.09$ (q, 1H, 3-H, $\left.\mathrm{J}_{3, \mathrm{Me}}=1.2\right), 7.52(\mathrm{~s}, 1 \mathrm{H}, 5-\mathrm{H})$. MS (m/z, \%): 246 (M+, 100), 218 (- CO, 20), 231 (- Me, 56), 203 (- Me, - CO, 27).

Anal. calc. for $\mathrm{C}_{14} \mathrm{H}_{14} \mathrm{O}_{4}$ : C 68.28, $\mathrm{H}$ 5.73; found: C 68.22, $\mathrm{H}$ 5.79.

\section{Synthesis of 4-methyl-6-R-furo[2,3-h]coumarins 19.}

Compound $18(0.00137 \mathrm{~mol})$, dioxane $(10 \mathrm{ml})$ and $20 \% \mathrm{H}_{2} \mathrm{SO}_{4}(5 \mathrm{ml})$ were heated at $50^{\circ} \mathrm{C}$ for 1 $\mathrm{hr}$ and poured then into water. The precipitate was filtered off and recrystallized from chloroform.

\section{6-Chloro-4-methylfuro[2,3-h]coumarin (19a).}

light yellow crystals, yield $=80 \%, \mathrm{mp} 209-210^{\circ} \mathrm{C} .1 \mathrm{H}$ NMR (acetone- $\left.\mathrm{d}_{6}, \delta, \mathrm{ppm} ; \mathrm{J}, \mathrm{Hz}\right): 2.57(\mathrm{~d}$, $\left.3 \mathrm{H}, 4-\mathrm{Me}, \mathrm{J}_{\mathrm{Me}, 3}=1.0\right), 6.35$ (q, 1H, 3-H, J $\left.3, \mathrm{Me}=1.0\right), 7.29$ (d, 1H, 9-H, J 9,8 $\left.=2.2\right), 7.77$ (s, 1H, 5$\mathrm{H}), 8.11\left(\mathrm{~d}, 1 \mathrm{H}, 8-\mathrm{H}, \mathrm{J}_{8,9}=2.2\right)$.

MS (m/z, \%): 234/236, (1CI), (M+, 97), 206/208, (1Cl), (-CO, 100), 205/207, (1Cl), (-HCO, 85), 171 (- CO, - CI, 37).

Anal. calc. for $\mathrm{C}_{12} \mathrm{H}_{7} \mathrm{ClO}_{3}$ : C 61.41, H 2.98; found: C 61.62, H 3.18. 


\section{6-Ethyl-4-methylfuro[2,3-h]coumarin (19b).}

light yellow crystals, yield $=83 \%$, mp $182-183^{\circ} \mathrm{C} .1 \mathrm{H} \mathrm{NMR}\left(\mathrm{DMSO}-\mathrm{d}_{6}, \delta, \mathrm{ppm} ; \mathrm{J}, \mathrm{Hz}\right): 1.36$ ( t, $3 \mathrm{H}, \mathrm{Me}, \mathrm{J}_{\mathrm{Me}, \mathrm{CH} 2}=7.4$ ), 2.54 (d, 3H, 4-Me, J Me,3 $=1.0$ ), 2.99 (q, 2H,- $\mathrm{CH}_{2^{-}}, \mathrm{J}_{-\mathrm{CH} 2-\mathrm{Me}}=7.4$ ), 6.26 $\left(\mathrm{q}, 1 \mathrm{H}, 3-\mathrm{H}, \mathrm{J}_{3, \mathrm{Me}}=1.0\right), 7.18\left(\mathrm{~d}, 1 \mathrm{H}, 9-\mathrm{H}, \mathrm{J}_{9,8}=2.2\right), 7.54(\mathrm{~s}, 1 \mathrm{H}, 5-\mathrm{H}), 7.99$ (d, 1H, 8-H, J 8,9 $=2.2$ ).

MS (m/z, \%): $228(\mathrm{M}+, 94), 200$ (- CO, 49), 185 (- CO, - Me, 100), 213 (- Me, 84).

Anal. calc. for $\mathrm{C}_{14} \mathrm{H}_{12} \mathrm{O}_{3}$ : C 73.68, H 5.26; found: C 73.28, H 5.13.

\section{Synthesis of acetoxyfurocoumarins $20 \mathrm{a}-\mathrm{j}, 23$.}

A substituted dihydrofurocoumarinone $(0.005 \mathrm{~mol})$, acetic anhydride $(20 \mathrm{ml})$ and potassium acetate $(0.0025 \mathrm{~mol})$ were heated at $120^{\circ} \mathrm{C}$ for $1 \mathrm{hr}$. The reaction mixture was filtered and excess of acetic anhydride was distilled off. The precipitate was filtered off and recrystallized from acetone.

\section{9'-Acetoxy-4-methylfuro[2,3-h]coumarin (20a).}

white crystals, yield $=70 \%, \mathrm{mp} 164-166^{\circ} \mathrm{C} .1 \mathrm{H} \mathrm{NMR}\left(\mathrm{CDCl}_{3}, \delta, \mathrm{ppm} ; \mathrm{J}, \mathrm{Hz}\right): 2.48(\mathrm{~s}, 3 \mathrm{H}, \mathrm{OAc})$, 2.49 (d, 3H, 4-Me, J Me,3 =0.9), 6.28 (q, 1H, 3-H, J $3, \mathrm{Me}=0.9$ ), 7.39 (d, 1H, 6-H, J 6,5=8.8), 7.54 $\left(\mathrm{d}, 1 \mathrm{H}, 5-\mathrm{H}, \mathrm{J}_{5,6}=8.8\right), 7.90(\mathrm{~s}, 1 \mathrm{H}, 8-\mathrm{H})$.

MS (m/z, \%): $258(\mathrm{M}+, 20), 216\left(-\mathrm{CH}_{2}=\mathrm{C}=\mathrm{O}, 100\right), 188\left(-\mathrm{CH}_{2}=\mathrm{C}=\mathrm{O},-\mathrm{CO}, 30\right), 187$ ($\left.\mathrm{CH}_{2}=\mathrm{C}=\mathrm{O},-\mathrm{HCO}, 10\right), 160\left(-\mathrm{CH}_{2}=\mathrm{C}=\mathrm{O},-2 \mathrm{CO}, 18\right)$.

Anal. calc. for $\mathrm{C}_{14} \mathrm{H}_{10} \mathrm{O}_{5}$ : C 65.12, H 3.90, ; found: C 65.32, H 3.80.

\section{9-Acetoxy-6-ethyl-4-methylfuro[2,3-h]coumarin (20b).}

white crystals, yield $=70 \%, \mathrm{mp} 213-214^{\circ} \mathrm{C} .1 \mathrm{H} \mathrm{NMR}\left(\mathrm{CDCl}_{3}, \delta\right.$, ppm; J, Hz): 1.36 (t, 3H, Me, J Me,-CH2- =7.4), 2.48 (s, 3H, OAc), 2.49 (d, 3H, 4-Me, J Me,3 =0.9), 2.94 (q, 2H, -CH2-, J -CH2-,Me $=7.4), 6.25$ (q, 1H, 3-H, J $3, \mathrm{Me}=0.9), 7.30(\mathrm{~s}, 1 \mathrm{H}, 5-\mathrm{H}), 7.88(\mathrm{~s}, 1 \mathrm{H}, 8-\mathrm{H})$.

MS (m/z, \%): $286(\mathrm{M}+, 15), 244\left(-\mathrm{CH}_{2}=\mathrm{C}=\mathrm{O}, 100\right), 229\left(-\mathrm{CH}_{2}=\mathrm{C}=\mathrm{O},-\mathrm{Me}, 14\right), 216$ ($\left.\mathrm{CH}_{2}=\mathrm{C}=\mathrm{O},-\mathrm{CO}, 7\right), 188\left(-\mathrm{CH}_{2}=\mathrm{C}=\mathrm{O},-2 \mathrm{CO}, 13\right)$.

Anal. calc. for $\mathrm{C}_{16} \mathrm{H}_{14} \mathrm{O}_{5}$ : C 67.13, $\mathrm{H} 4.93$, ; found: C 67.42, $\mathrm{H} 4.90$. 


\section{9'-Acetoxy-8-chloro-4-methylfuro[2,3-h]coumarin (20c).}

white crystals, yield $=70 \%, \mathrm{mp} 210-211^{\circ} \mathrm{C} .1 \mathrm{H} \mathrm{NMR}\left(\mathrm{CDCl}_{3}, \delta, \mathrm{ppm} ; \mathrm{J}, \mathrm{Hz}\right): 2.51$ (s, 3H, OAc), $2.46\left(\mathrm{~d}, 3 \mathrm{H}, 4-\mathrm{Me}, \mathrm{J}_{\mathrm{Me}, 3}=0.9\right), 6.25\left(\mathrm{q}, 1 \mathrm{H}, 3-\mathrm{H}, \mathrm{J}_{3, \mathrm{Me}}=0.9\right), 7.32\left(\mathrm{~d}, 1 \mathrm{H}, 6-\mathrm{H}, \mathrm{J}_{6,5}=8.8\right), 7.50$ $\left(\mathrm{d}, 1 \mathrm{H}, 5-\mathrm{H}, \mathrm{J}_{5,6}=8.8\right)$.

MS (m/z, \%): 292/294 (1Cl) (M+, 17), 250/252 (1Cl) $\left(-\mathrm{CH}_{2}=\mathrm{C}=\mathrm{O}, 100\right), 222 / 224\left(-\mathrm{CH}_{2}=\mathrm{C}=\mathrm{O}\right.$, $\mathrm{CO}, 9), 221 / 223(1 \mathrm{Cl})\left(-\mathrm{CH}_{2}=\mathrm{C}=\mathrm{O},-\mathrm{HCO}, 8\right)$.

Anal. calc. for $\mathrm{C}_{14} \mathrm{H}_{9} \mathrm{ClO}_{5}$ : C 57.40, $\mathrm{H} 3.07, \mathrm{Cl} 12.13$; found: C 57.32, H 3.03, Cl 12.18.

\section{9'-Acetoxy-8-bromo-4-methylfuro[2,3-h]coumarin (20d).}

white crystals, yield $=75 \%$, mp $225-227^{\circ} \mathrm{C} .1 \mathrm{H} \mathrm{NMR}\left(\mathrm{CDCl}_{3}, \delta\right.$, ppm; J, Hz): 2.51 (s, 3H, OAc), 2.46 (d, 3H, 4-Me, J Me,3 =0.9), 6.25 (q, 1H, 3-H, J $3, \mathrm{Me}=0.9), 7.34$ (d, 1H, 6-H, J 6,5 =8.8), 7.49 $\left(\mathrm{d}, 1 \mathrm{H}, 5-\mathrm{H}, \mathrm{J}_{5,6}=8.8\right)$.

MS (m/z, \%): 336/338 (1Br) (M+, 12), 294/296 (1Br) $\left(-\mathrm{CH}_{2}=\mathrm{C}=\mathrm{O}, 100\right), 266 / 268$ (1Br) ($\left.\mathrm{CH}_{2}=\mathrm{C}=\mathrm{O},-\mathrm{CO}, 7\right), 238 / 240(1 \mathrm{Br})\left(-\mathrm{CH}_{2}=\mathrm{C}=\mathrm{O},-2 \mathrm{CO}, 23\right), 159\left(-\mathrm{CH}_{2}=\mathrm{C}=\mathrm{O},-2 \mathrm{CO},-\mathrm{Br}, 33\right)$. Anal. calc. for $\mathrm{C}_{14} \mathrm{H}_{9} \mathrm{BrO}_{5}$ : C 49.83, $\mathrm{H} 2.67, \mathrm{Br} 23.70$; found: $\mathrm{C} 49.88, \mathrm{H} 2.69, \mathrm{Br} 23.70$.

\section{9'-Acetoxy-8-acetyl-4-methylfuro[2,3-h]coumarin (20h).}

white crystals, yield $=65 \%, \mathrm{mp} 195-196^{\circ} \mathrm{C} .1 \mathrm{H} \mathrm{NMR}\left(\mathrm{CDCl}_{3}, \delta\right.$, ppm; J, Hz): $2.58(\mathrm{~s}, 3 \mathrm{H}, \mathrm{OAc})$, 2.49 (d, 3H, 4-Me, J Me,3 =0.9), 2.59 (s, 3H, Ac), 6.29 (q, 1H, 3-H, J 3,Me =0.9), 7.45 (d, 1H, 6-H, $\left.\mathrm{J}_{6,5}=8.8\right), 7.70\left(\mathrm{~d}, 1 \mathrm{H}, 5-\mathrm{H}, \mathrm{J}_{5,6}=8.8\right)$.

MS (m/z, \%): $300(\mathrm{M}+, 7), 258\left(-\mathrm{CH}_{2}=\mathrm{C}=\mathrm{O}, 100\right), 230\left(-\mathrm{CH}_{2}=\mathrm{C}=\mathrm{O},-\mathrm{CO}, 20\right), 243$ $\mathrm{CH}_{2}=\mathrm{C}=\mathrm{O}$, - Me, 39), $215\left(-\mathrm{CH}_{2}=\mathrm{C}=\mathrm{O},-\mathrm{Me}-\mathrm{C}=\mathrm{O}, 9\right)$.

Anal. calc. for $\mathrm{C}_{16} \mathrm{H}_{12} \mathrm{O}_{6}$ : C 64.00, H 4.03; found: C 64.17, H 3.93. 
8-(4'-Methyldihydrofuro[2',3'-h]coumarin-9'-one-8'-methylideno) -4-methyldi hydrofuro[2,3h]coumarin-9-one (21).

Compound $7 \mathrm{~g}(0.002 \mathrm{~mol})$ and acetic anhydride $(20 \mathrm{ml})$ were heated at $130^{\circ} \mathrm{C}$ for $2 \mathrm{hrs}$. The precipitate was filtered off and washed with hot acetone. Compound 21, yellow crystals, yield $=45 \%, \mathrm{mp} 308^{\circ} \mathrm{C}$ (decomp.). 1H NMR (DMSO-d 6 , $\left.\delta, \mathrm{ppm} ; \mathrm{J}, \mathrm{Hz}\right): 2.55$ (d, 3H, 4-Me, J Me,3 =1.2), 2.57 (d, 3H, 4'-Me, J Me,3' =0.9), 6.29 (q, 1H, 3-H, J 3,Me =1.2), 6.44 (q, 1H, 3'- $\mathrm{H}, ~ J_{3}$ ',Me $=0.9), 6.96(\mathrm{~s}, 1 \mathrm{H},=\mathrm{C}-\mathrm{H}), 7.49\left(\mathrm{~d}, 1 \mathrm{H}, 6-\mathrm{H}, \mathrm{J}_{6,5}=9.0\right), 7.67\left(\mathrm{~d}, 1 \mathrm{H}, 6^{\prime}-\mathrm{H}_{,} \mathrm{J}_{6}, 5^{\prime}=8.8\right), 7.91$ (d, $\left.1 \mathrm{H}, 5^{\prime}-\mathrm{H}, \mathrm{J}_{5}, 6^{\prime}=8.8\right), 8.23\left(\mathrm{~d}, 1 \mathrm{H}, 5-\mathrm{H}_{1} \mathrm{~J}_{5,6}=9.0\right)$.

MS (m/z, \%): 442 (M+, 100), 425(- OH, 10), 414(- CO, 5), 385 (- CO, -HCO, 7).

Anal. calc. for $\mathrm{C}_{25} \mathrm{H}_{14} \mathrm{O}_{8}$ : C 67.88, H 3.19; found: C 67.93, $\mathrm{H} 3.28$

\section{4-Methylfuro[2,3-g]coumarin (22).}

Compound $3 \mathrm{~b}(0.001 \mathrm{~mol}), \mathrm{NaBH}_{4}(0.0011 \mathrm{~mol})$ and ethanol $(7 \mathrm{ml})$ were stirred at room temperature for $2 \mathrm{hrs}$. The mixture was then acidified by phosphorous acid and heated under boiling for $1 \mathrm{hr}$. The precipitate was filtered off and recrystallized from ethanol. Compound 22, white crystals, yield $=65 \%, \mathrm{mp} 200^{\circ} \mathrm{C} .1 \mathrm{H} \mathrm{NMR}\left(\mathrm{DMSO}_{6}, \delta, \mathrm{ppm} ; \mathrm{J}, \mathrm{Hz}\right): 2.49$ (d, 3H,4-Me, J Me,3=1.1), 6.43 (q, 1H, 3-H, $\left.\mathrm{J}_{3, \mathrm{Me}}=1.1\right), 7.07\left(\mathrm{dd}, 1 \mathrm{H}, 6-\mathrm{H}, \mathrm{J}_{6,7}=2.5, \mathrm{~J}_{6,5}=0.8\right), 7.67(\mathrm{~d}, 1 \mathrm{H}, 5-\mathrm{H}$, $\left.\mathrm{J}_{5,6}=0.8\right), 8.07(\mathrm{~s}, 1 \mathrm{H}, 9-\mathrm{H}), 8.08\left(\mathrm{~d}, 1 \mathrm{H}, 7-\mathrm{H}, \mathrm{J}_{7,6}=2.5\right)$.

MS (m/z, \%): $200(\mathrm{M}+, 100)$.

Anal. calc. for $\mathrm{C}_{12} \mathrm{H}_{8} \mathrm{O}_{3}$ : C 72.00, $\mathrm{H} 4.03$; found: C 71.79, $\mathrm{H} 3.88$.

\section{6-Acetoxy-4,9-dimethylfuro[2,3-g]coumarin (23).}

white crystals, yield $=73 \%$, mp 195-196 ${ }^{\circ} \mathrm{C} .1 \mathrm{H} \mathrm{NMR}\left(\mathrm{CDCl}_{3}, \delta\right.$, ppm; J, Hz): 2.42 (s, 3H, 9Me), 2.52 (d, 3H, 4-Me, $\mathrm{J}_{\mathrm{Me}, 3}=1.2$ ), 2.59 (s, 3H, OAc),6.28 (q, 1H, 3-H, J 3,Me $=1.2$ ), 7.62 (s, 1H, 5-H,), $8.11(\mathrm{~s}, 1 \mathrm{H}, 7-\mathrm{H})$.

$\mathrm{MS}(\mathrm{m} / \mathrm{z}, \%): 272(\mathrm{M}+, 24), 230\left(-\mathrm{CH}_{2}=\mathrm{C}=\mathrm{O}, 100\right), 202\left(-\mathrm{CH}_{2}=\mathrm{C}=\mathrm{O},-\mathrm{CO}, 22\right), 201(-$ $\left.\mathrm{CH}_{2}=\mathrm{C}=\mathrm{O},-\mathrm{HCO}, 15\right)$.

Anal. calc. for $\mathrm{C}_{14} \mathrm{H}_{10} \mathrm{O}_{5}$ : C 65.12, H 3.90; found: C 65.39, H 3.88. 


\section{4-Methyl-7-(4'-methoxyphenylazo)dihydrofuro[2,3-g]coumarin (24).}

This compound has been prepared similarly to preparation of the compounds 13

Red crystals, yield $=70 \%, \operatorname{mp} 280-282^{\circ} \mathrm{C} .1 \mathrm{H} \mathrm{NMR}\left(\mathrm{CDCl}_{3}, \delta\right.$, ppm; J, Hz): 2.48 (d, 3H,4-Me, J Me,3=1.1), 3.81 (s, 3H, 4'-OMe), 6.31 (q, 1H, 3-H, J 3,Me=1.1), 6.92 (d, 2H, 3'-H, 5'-H, J 3', '= $\left.5^{\prime}, 6^{,}=9.0\right), 7.22\left(\mathrm{~d}, 2 \mathrm{H}, 2^{\prime}-\mathrm{H}, 6^{\prime}-\mathrm{H}, \mathrm{J}_{2}{ }^{,}, 3^{\prime}=\mathrm{J}_{6},{ }_{5},=9.0\right), 8.12(\mathrm{~s}, 1 \mathrm{H}, 5-\mathrm{H}), 8.33$ (s, 1H, NH).

MS (m/z, \%): $350(\mathrm{M}+, 100), 335(-\mathrm{Me}, 7.3), 203\left(-4-\mathrm{MeOC}_{6} \mathrm{H}_{4}-\mathrm{N}==\mathrm{N}=\mathrm{C}, 12\right), 174$ (- 4$\left.\mathrm{MeOC}_{6} \mathrm{H}_{4}-\mathrm{N}==\mathrm{N}=\mathrm{C},-\mathrm{HCO}, 10\right)$.

Anal. calc. for $\mathrm{C}_{19} \mathrm{H}_{14} \mathrm{~N}_{2} \mathrm{O}_{5}$ : C 65.14, $\mathrm{H} 4.03$; $\mathrm{N}$ 8.00; found: $\mathrm{C} 65.22, \mathrm{H} 4.31, \mathrm{~N}$ 8.31.

\section{References}

1. Murray, R. D. H. The Natural Coumarins, occurrence, chemistry and biochemistry.; WileyInterscience: New York, 1982.

2. Fahr, E. Pharmazeutishe Zeitung 1982, 127, 163.

3. Edelson, R. L. J. Photochem. Photobiol., B: Biol. 1991, 10, 165.

4. Guiotto, A.; Chilin, A.; Manzini, P.; Dall'Aqua, F.; Bordin, F.; Rodighiero, P. IL Farmaco 1995, 50, 479 .

5. Saffran, W. A. In Psoralen DNA Photobiology; Gasparro, F. P., Ed.; CRC Press: INC.: Boca Raton, Fl, 1988; Vol. 11, Ch. 6, p 73.

6. Dall'Acqua, F.; Vedaldi, D.; Caffieri, S.; Guiotto, A.; Rodighiero, P.; Carrlassare, F.; Bordin, F. J. Med. Chem. 1981, 24, 178.

7. Guiotto, A.; Rodighiero, P.; Manzini, P.; Pfstorini, G.; Carlassare, F.; Vedaldi, D. et al. J. Med. Chem. 1984, 27, 959.

8. Bordin, F.; Carlassare, F.; Baccichetti, F.; Guiotto, A.; Rodighiero, P.; Vedaldi, D.; Dall'Acqua, F. Photochem. Photobiol. 1979, 29, 1063.

9. Dall'Acqua, F.; Vedaldi, D.; Guiotto, A.; Rodighiero, P.; Carlassare, F.; Baccichetti, F.; Bordin, F. J. Med. Chem. 1981, 24, 806. 
10. Guiotto, A.; Rodighiero, P.; Pastorini, G.; Manzini, P.; Bordin, F.; Baccichetti, F.; Carlassare, F.; Vedaldi, D.; Dall'Acqua, F. Eur. J. Med. Chem-Chim. Ther. 1981, 16, 489.

11. Dall'Acqua, F.; Vedaldi, D.; Bordin, F.; Baccichetti, F.; Carlassare, F.; Tamaro, M.; Rodighiero, P.; Pastorini, G.; Guiotto, A.; Recchia, G.; Cristofolini, M. J. Med. Chem. 1983, 26,870 .

12. Vedaldi, D.; Dall'Acqua, F.; Baccichetti, F.; Carlassare, F.; Bordin, F.; Rodighiero, P.; Manzini, P.; Guiotto, A. Farmaco 1991, 46, 1381.

13. Demaret, J.-P.; Brunie, S.; Ballini, J.-P.; Cadet, J.; Vigny, P. J. Photochem. Photobiology B: Biol. 1990, 6, 207.

14. Caffieri, S.; Vedaldi, D.; Chilin, A.; Pozzan, A. J. Photochem. Photobiology B: Biol. 1994, $22,151$.

15. Chen, X.; Kagan, J. J. Photochem. Photobiology B: Biol. 1994, 23, 27.

16. Csik, G.; Ronto, G.; Nocentini, S.; Averbeck, S.; Averbeck, D. J. Photochem. Photobiology B: Biol. 1994, 24, 129.

17. Chen, X.; Kagan J. J. Photochem. Photobiology B: Biol. 1994, 22, 51.

18. Chilin, A.; Marzano, C.; Guiotto, A.; Manzini, P.; Baccichetti, F.; Carlassare, F.; Bordin, F. J. Med. Chem. 1999, 42, 2936.

19. Dallavia, L.; Gia, O.; Magno, S. M.; Santana, L.; Teijeira, M.; Uriarte, E. J. Med. Chem. 1999, 42, 4405.

20. Traven, V. F.; Rozhkov, R. V.; Tolmachev, A. Yu.; Kuznezova, N. A.; Podhaluzina, N. Ya.; Carberry, E. A. Heterocyclic Commun. 1997, 3, 4.

21. Traven, V. F.; Kravtchenko, D. V.; Chibisova, T. A. Mendeleev Commun., 1995, 21.

22. Traven, V. F.; Kravtchenko, D. V.; Chibisova,T. A.; Shorshnev, S. V.; Eliason, R.; Wakefield, D. H. Heterocyclic Commun., 1996, 2, 345.

23. Kravtchenko, D. V.; Traven, V. F.; Chibisova, T. A. Heterocyclic Commun.1997, 3, 331.

24. Traven, V. F.; Kravtchenko, D. V.; Chibisova, T. A. Mendeleev Commun. 1997, 249.

25. Traven, V. F.; Saharuk, I. I.; Kravtchenko, D. V. Heterocyclic Commun. 1998, 4, 429.

26. Traven, V. F.; Saharuk, I. I.; Kravtchenko, D. V.; Makarov, I. G. Heterocyclic Commun. 1999, 5, 379.

27. Traven, V. F.; Saharuk, I. I.; Kravtchenko, D. V. Khimija Geteroziklicheskih Soedinenii, submitted, 1999. 
28. Kravtchenko, D. V.; Chibisova, T. A.; Traven, V. F. Zh. Org. Khim. 1999, 35, 924. 